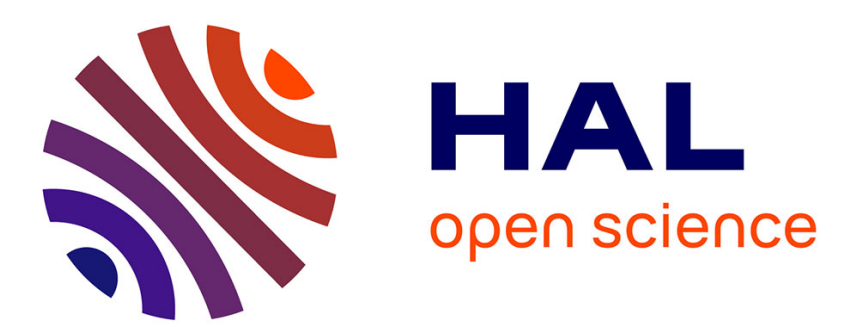

\title{
From winter to summer and back: Lessons from the parameterization of a seasonal food web model for the Bialowieża forest
}

\author{
Alix Sauve, Frederic Barraquand
}

\section{- To cite this version:}

Alix Sauve, Frederic Barraquand. From winter to summer and back: Lessons from the parameterization of a seasonal food web model for the Bialowieża forest. Journal of Animal Ecology, 2020, 89 (7), pp.1628-1644. 10.1111/1365-2656.13227 . hal-03001021

\section{HAL Id: hal-03001021 \\ https://hal.science/hal-03001021}

Submitted on 14 Dec 2020

HAL is a multi-disciplinary open access archive for the deposit and dissemination of scientific research documents, whether they are published or not. The documents may come from teaching and research institutions in France or abroad, or from public or private research centers.
L'archive ouverte pluridisciplinaire HAL, est destinée au dépôt et à la diffusion de documents scientifiques de niveau recherche, publiés ou non, émanant des établissements d'enseignement et de recherche français ou étrangers, des laboratoires publics ou privés. 


\title{
From winter to summer and back: lessons from the
}

\author{
parameterisation of a seasonal food web model for the Białowieża \\ forest
}

\author{
Alix M. C. Sauve*1 and Frederic Barraquand ${ }^{1,2}$ \\ ${ }^{1}$ University of Bordeaux, Integrative and Theoretical Ecology, LabEx COTE, France \\ ${ }^{2}$ CNRS, Institute of Mathematics of Bordeaux, France
}

This manuscript is published in Journal of Animal Ecology. Cite as:

Alix M.C. Sauve, Frédéric Barraquand, From winter to summer and back: lessons from the parameterisation of a seasonal food web model for the Bialowieza forest, Journal of Animal Ecology, 2020. https://doi.org/10.1111/1365-2656.13227

\footnotetext{
*Corresponding author: alix.sauve@cri-paris.org
} 


\begin{abstract}
1. Dynamic food web models describe how species abundances change over time as a function of trophic and life-history parameters. They are essential to predicting the response of ecosystems to perturbations. However, they are notoriously difficult to parameterise, so that most models rely heavily either on allometric scaling of parameters or inverse estimation of biomass flows. The allometric approach makes species of comparable body mass have near-identical parameters which can generate extinctions within a trophic level. The biomass flow approach is more precise, but is restricted to steady-states, which is not appropriate for time-varying environments.
\end{abstract}

2. Adequately parameterising large food webs of temperate and arctic environments requires dealing both with many species of similar sizes and a strongly seasonal environment. Inspired by the rich empirical knowledge on the vertebrate food web of the Białowieża forest, we parameterise a bipartite food web model comprising 21 predators and 124 prey species. Our model is a nonautonomous coupled ODE (Ordinary Differential Equations) system that allows for seasonality in life-history and predation parameters.

3. Birth and death rates, seasonal descriptions of diet for each species, food requirements and biomass information are combined into a seasonal parameterisation of a dynamic food web model. Food web seasonality is implemented with time-varying intrinsic growth rate and interaction parameters, while predation is modelled with both type I and type II functional responses.

4. All our model variants allow for $>80 \%$ persistence in spite of massive apparent competition, and a quantitative match to observed (seasonal) biomasses. We also identify trade-offs between maximising persistence, reproducing observed biomasses, and ensuring model robustness to sampling errors. Although multi-annual cycles are expected with type II functional responses, they are here prevented by a strong predator self-regulation. We discuss these results and possible improvements of the model. 
5. We provide a general workflow to parameterise dynamic food web models in seasonal environments, based on a real case study. This may help to better predict how biodiverse food webs respond to changing environments.

Keywords: bipartite food web; Białowieża forest; seasonality; parameterisation; predator-prey dynamics; Lotka-Volterra model; periodic forcing.

\section{Introduction}

Model parameterisation is fundamental to ensure prediction accuracy of population dynamics (be the prediction qualitative or quantitative). Even simple models of predator-prey dynamics, modelling transfers of biomass from the prey to the predator alongside prey intrinsic growth and predator mortality, are quite challenging to parameterise. This is partly because model outcomes can be quite sensitive to the value of the parameters themselves. For instance, in the RosenzweigMacArthur model describing a two-dimensional predator-prey community, already 5 parameters interact in determining the dynamical behaviour of the community (Bazykin, 1998). Options to tackle this "plague of parameters" (as coined by Yodzis \& Innes, 1992) range from randomly sampling the parameter space, which provides little predictive power, to a detailed accounting of each process rate, which is very demanding in empirical data on the modelled ecosystem (e.g., Hanski \& Korpimäki, 1995; Turchin \& Hanski, 1997; Gilg et al., 2003). Sometimes, despite the best efforts of the research community, some of the parameters still have to be "guesstimated". An alternative approach, pioneered by Yodzis \& Innes (1992), relies on scaling model parameters as a function of species body masses and metabolic types, which considerably reduces the amount of empirical data

necessary to mimic realistic systems (see also Weitz \& Levin, 2006, for a reframed version of the Rosenzweig-MacArthur model). 
Moving from two-species predator-prey systems to many-species food webs considerably aggravates the parameterisation challenge. Allometric scaling of parameters then offers a parsimonious, and thus tractable, solution to this challenge (Williams et al., 2007; Hudson \& Reuman, 2013). Allometric scaling of food webs has been successful in explaining species coexistence and community stability (e.g., Brose et al., 2006; Kartascheff et al., 2010) and in predicting the strength of trophic cascades (from predators to resources) as a function of predator body size (e.g., Schneider et al., 2012; DeLong et al., 2015). With practical applications in mind, allometric trophic network models have the potential to reproduce the dynamics of food webs with about a dozen of compartments observed in time-varying environments (e.g., Boit et al., 2012; Curtsdotter et al., 2019). However, turning to even larger food webs, where many species can have the same body mass, highlights an issue with the allometric approach: by construction, predation on two species or compartements with similar body masses will be parameterised alike. Consequently, if there are many species with similar body masses, and they share similar food sources or predators, they will tend to exclude each other out in Lotka-Volterra or similar systems. Tuning the strength of trophic relationship with other traits (e.g., species mobility, Brose et al., 2019) or prey availability (e.g., Kalinkat et al., 2011; Quévreux \& Brose, 2019) could help to avoid this outcome, but at the cost of increased data requirements.

The popular, empirically-driven alternative to purely-allometric parameterisations uses an estimated biomass flow approach (Ulanowicz \& Kay, 1991; Christensen \& Pauly, 1992; de Ruiter et al., 1995). In the biomass flow approach, one quantifies the amount of biomass transiting between compartements of the modelled ecosystem, as well as exchanges with the outside world (migration, harvesting). However, biomass flow approaches rely on the assumption of mass-balance (or energy-balance) within each compartment (Gauzens et al., 2019). Although enabling parameter 
estimation, this working hypothesis is somewhat problematic to model time-varying ecosystems, such as strongly seasonal ecosystems. Not only seasonal ecosystems have rates of change where biomass gains do not always equilibrate with losses, but the very existence of the seasonal forcing may induce dynamics much more complex than a single equilibrium point (Rinaldi et al., 1993; Taylor et al., 2013). For that reason, empirically-based food web studies on time-varying environments using a steady-state assumption usually reduce their system to a succession of steady-states, each corresponding to a given time slice, be it the year (e.g., Legagneux et al., 2012; Chevillot et al., 2019) or the season (e.g., Saavedra et al., 2016). The main limitation of this "succession trick" is that the dynamics of each time slice are considered to be independent.

Here, we use a modelling philosophy similar to that of studies which parameterised seasonal predator-prey systems with two to four species (e.g., Hanski \& Korpimäki, 1995; Turchin \& Hanski, 1997; Gilg et al., 2003), using a mixture of seasonal life-history data, some allometric relationships, and attack rates reconstructed from seasonal empirical data. However, we apply this approach to a much larger seasonal food web, the terrestrial vertebrate food web of the Białowieża forest (Jędrzejewska \& Jȩdrzejewski, 1998). By focusing on the Białowieża forest as a case study, we benefit from a decade of detailed monitoring on a diverse multitrophic community, describing intra-annual variations of species densities and seasonal predator diets. Essentially, we generalise classic seasonal predator-prey models with time-dependent parameters to food webs. This could be construed as a blend between the theoretically-driven allometric/life-history approaches and the empirically-driven biomass flow approaches. We specify a parametrisation workflow for both type I and type II functional responses of predators, and two different ways of estimating predator regulation. In the following, we first describe how the network changes from winter to summer in the Białowieża forest, and in a second step, we parameterise the dynamical food web model based 
on the data compiled in Jẹdrzejewska \& Jȩdrzejewski (1998)'s monograph. We then simulate the dynamics of the different versions of the model food web and finally confront model outcomes to the observed data, using multiple diagnostics (species biomasses, winter-to-summer transition, species abundance curves).

\section{The seasonal predator-prey community of the Białowieża forest}

We chose the food web of the Białowieża forest (Poland and Belarus) as an inspiration for our study. Our reasons are three-fold. First, the structural stability approach for separated seasons of Saavedra et al. (2016), applied to the Białowieża forest, motivated us to investigate the dynamics of this ecosystem under seasonal forcing, rather than steady-state per season. Second, there is ample additional biological information due to extensive monitoring (Jȩdrzejewska \& Jȩdrzejewski, 1998). This creates opportunities to shift from theoretical analyses, assuming only feasibility of stable fixed points, to fully parameterised models that better represent the actual set of life-history constraints on that community. Third, this ecosystem is characterised by a continental climate with marked differences between summer and winter processes, which can produce complex dynamics (Rinaldi et al., 1993; Turchin \& Hanski, 1997). For a detailed description of the Białowieża forest, with an impressive description of the data gathered over the years, we refer the reader to Jȩdrzejewska \& Jȩdrzejewski (1998)'s authoritative book.

The food web, combining data collected between 1985 and 1996 in the Polish part of the Białowieża forest, is composed by 142 taxa: 77 birds, 41 mammals, 14 fishes, 6 amphibians, 3 reptiles, and 1 crustacean. We also consider 3 additional groups (carcasses, earth worms, and Hymenoptera) which represent substantial resources to some predators. In total, the food web describes 501 trophic relationships between 21 predators and 124 food sources. 
One can consider two distinct time slices for this food web: one for summer (from 16th April to 30th September, i.e. 168 days), and one for winter (from 1st October to 15th April, i.e. 197 days). These two broadly defined seasons differ in two ways: species composition (due to species migration and dormancy), and interaction composition (due to predator dietary shifts following prey availability). Eventually, summer and winter share 17 predators, 72 food sources, and 235 trophic interactions. The food web is overall more diverse and the number of interactions higher during summer (136 species, 441 interactions) than during winter (101 species, 292 interactions).

\subsection{Estimation of predators' intakes of prey}

In their comprehensive book, Jȩdrzejewska \& Jȩdrzejewski (1998) provide substantial information about predation in the Białowieża forest. The empirical data compiled in this monograph cover predator diets for the two broad seasons we defined above, and enable us to estimate predator intakes for each season. Diet compositions are based on the analysis of predators' pellets and scats which then provide effective biomass proportions of prey items in each predatory species diet. Herein, we calculate predator intakes of each prey group to eventually estimate the direct impact of each pairwise predatory link in the food web during an average year.

Hereafter, $G_{k i}$ denotes the intake of prey species $k$ by an individual predator $i$ over an average year (in g. $\mathrm{N}^{-1}$ ). Basing our calculation on the physiological needs of predators (following Jẹdrzejewska \& Jȩdrzejewski, 1998), we calculate the annual intake of prey $k$ by one predator $i$ as:

$$
G_{k i} \simeq(D F I)_{i} \times\left(\omega_{k i}^{S} \times\left(n_{\text {days }}\right)_{i}^{S}+\omega_{k i}^{W} \times\left(n_{\text {days }}\right)_{i}^{W}\right)
$$

where $(D F I)_{i}$ is the daily food intake of predator $i$ (in mass per day per individual), and $\omega_{k i}^{S}$ and $\omega_{k i}^{W}$ the biomass fractions of prey $k$ in predator $i$ 's diet during summer and winter respectively. 
$\left(n_{\text {days }}\right)_{i}^{S}$ and $\left(n_{\text {days }}\right)_{i}^{W}$ are the number of days of predator $i$ 's presence in the Białowieża forest during summer and winter respectively. Recovering seasonal intakes $G_{k i}^{S}$ and $G_{k i}^{W}$ is done by considering season-specific terms in eq. 1 to estimate each.

Data on predator diets suggest multiple links to other predator species, which were ignored in the bipartite food web presented in the previous modelling of the Białowieża forest by Saavedra et al. (2016, using structural approaches without paramerisation). Identifying whether these predatorpredator links correspond to active hunting, interference or scavenging is not possible with this type of data (Jȩdrzejewska \& Jȩdrzejewski, 1998). We thus chose to focus on trophic links between two trophic levels (as in Saavedra et al., 2016). The resulting seasonal food web is displayed in Fig. 1. 


\section{Summer}

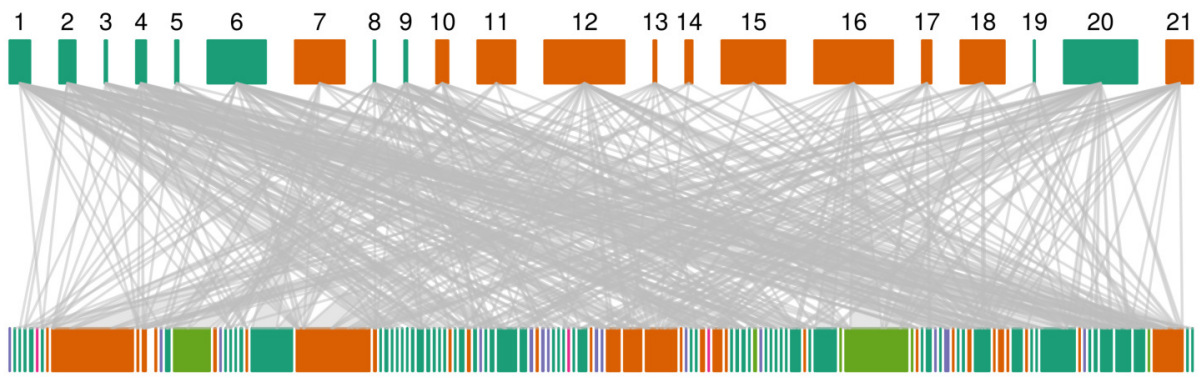

\section{Winter}

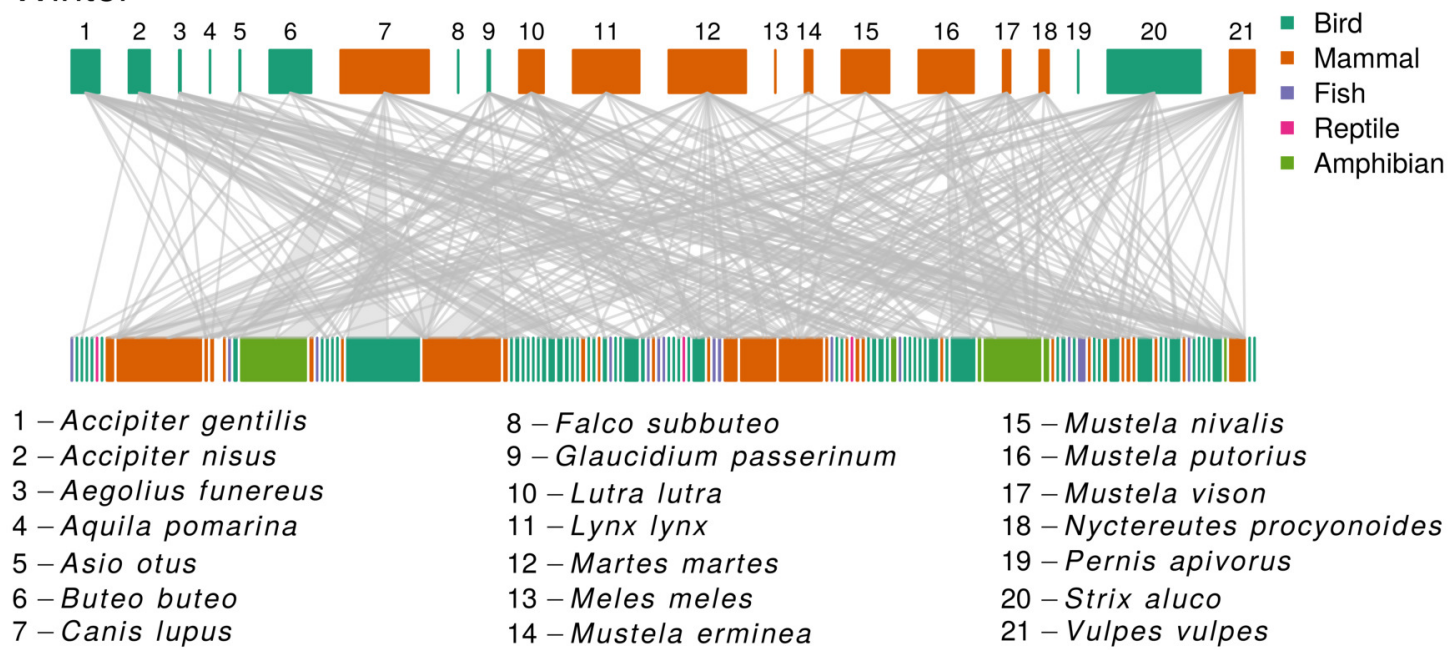

Figure 1: The food web in the Białowieża forest during summer and winter. Upper boxes correspond to predators, and lower boxes to prey species. Colours match species groups, and edge widths are proportional to the quantity eaten by each predator population on each prey population (in g.ha ${ }^{-1}$ ) during each season. The size of upper boxes is proportional to the predator's total removal of prey, and the size of lower boxes is proportional to the amount of prey eaten by all predator species combined. We do not represent interactions involving invertebrates and carcasses for the sake of clarity, although we model quantities eaten on these compartments.

\subsection{Metrics for describing seasonal variations within food web}

To better understand how food web seasonality may contribute to community dynamics, and whether it is worth considering such extra complexity, we need to grasp how the quantitative structure of the food web varies between seasons. Trophic links are quantified as prey biomass harvested by predator populations during each season. In other words, each trophic link is seasonally 
weighted with $\Gamma_{k i}^{z}=G_{k i}^{z} \times\left(C_{i} / M_{i}\right)_{z}$ where $G_{k i}^{z}$ is the per-capita intake of prey $k$ by the predator species $i$ (in g.ha- $\left.\mathrm{A}^{-1} \cdot \mathrm{N}^{-1}\right)$ during season $z$, and $\left(C_{i} / M_{i}\right)_{z}$ is the density of the predator $i$ during season $z$ (in N.ha $\left.{ }^{-1}\right)$. We investigate structural changes with two questions.

Are trophic fluxes seasonal? We quantify predation seasonality with the following index for predator $j:$

$$
P S_{j}=1+\sum_{z \in\{S, W\}}\left(\frac{\Gamma_{\bullet j}^{z}}{\Gamma_{\bullet j}^{S}+\Gamma_{\bullet j}^{W}} \ln \left(\frac{\Gamma_{\bullet j}^{z}}{\Gamma_{\bullet j}^{S}+\Gamma_{\bullet j}^{W}}\right)\right)
$$

where $\Gamma_{\bullet j}^{z}=\sum_{h} \Gamma_{h j}^{z}$ is the quantity of prey eaten by the predator $j$ population during season $z$. To adapt this index to prey species, this quantity should be replaced with $\Gamma_{j \bullet}^{z}=\sum_{h} \Gamma_{j h}^{z}$, the quantity of prey $j$ eaten by the predator community during season $z$. Values near 1 indicate highly seasonal predation while lower values suggest more constant predation from the perspective of species $j$. For predators, $P S_{j}$ reflects whether their hunting activity in the Białowieża forest is constant across seasons. From the prey perspective, it rather translates whether they undergo constant predation pressure.

How does predation differ between summer and winter? First, we quantify the trophic similarity between seasons of each species $j$ with the Jaccard index (qualitative similarity) and the Bray-Curtis index (quantitative similarity of estimated impact $\Gamma_{k i}^{z}$ on prey $k$ ). Second, we evaluate predator generality and prey vulnerability as proportional generality (see R package bipartite; Dormann et al., 2009) during each season $z$, and compare them for each species. Proportional generality is the effective number of predators/prey $e^{H_{j}^{z}}$ of species $j$ divided by the maximum potential number of predators/prey $e^{H_{\max }^{z}}$. For a given season $z$, we calculate $H_{j}^{z}$ and $H_{\max }^{z}$ as

$$
\left\{\begin{array}{c}
H_{j}^{z}=-\sum_{k} \frac{\Gamma_{k j}^{z}}{\Gamma_{\bullet j}^{z}} \ln \left(\frac{\Gamma_{k j}^{z}}{\Gamma_{\bullet j}^{z}}\right) \\
H_{\text {max }}^{z}=-\sum_{k} \frac{R_{k}^{z}}{R_{\bullet}^{z}} \ln \left(\frac{R_{k}^{z}}{R_{\bullet}^{z}}\right)
\end{array}\right.
$$


if species $j$ is a predator. For prey species, indices are swapped, and we use predator biomass densities $C_{i}^{z}$ during season $z$ instead of prey biomass densities $R_{k}^{z}$ (seasonal densities are available in Supporting Information A). This method allows us to compare species generality or vulnerability while taking into account that the amount of predators or prey varies between seasons.

\subsection{Effects of seasonality on the structure of the food web of the Białowieża forest}

Predators (all but Asio otus and Nyctereutes procyonoides) fall into two well-identified groups: year-round predators (with low $P S_{i}$ ), and seasonal predators (with high $P S_{i}$ ). Prey species show a less clear-cut distribution as many species undergo an intermediate level of predation seasonality (Fig. 2A). We also note that predators harvesting prey equally between seasons and prey undergoing equal predation between seasons (in which cases $P S_{i}=1-\ln (2) \simeq 0.307$ ) are few. This is likely due to winter lasting a longer time (197 days against 168) thereby the winter food web exhibits more important trophic fluxes.

Many predators tend to feed on different prey between summer and winter (Fig. 2B). The Jaccard index indicates that diet composition changes between summer and winter, although a fifth of prey species are attacked by exactly the same set of predators. Quantifying predator intakes within each season suggests more important changes in their diets: part of their diet composition changes (Fig. 2B, open circles), and their predation effort also tends to shift to different prey species between summer and winter (Fig. 2B, closed circles).

Finally, prey species appear equally vulnerable during winter and summer (consistent with Wilcoxon's test; $W=1421, p=0.43$; Fig. $2 \mathrm{C}$ ) while predators become more specialised during winter $(W=114, p<0.01$, Fig. $2 \mathrm{C})$. 

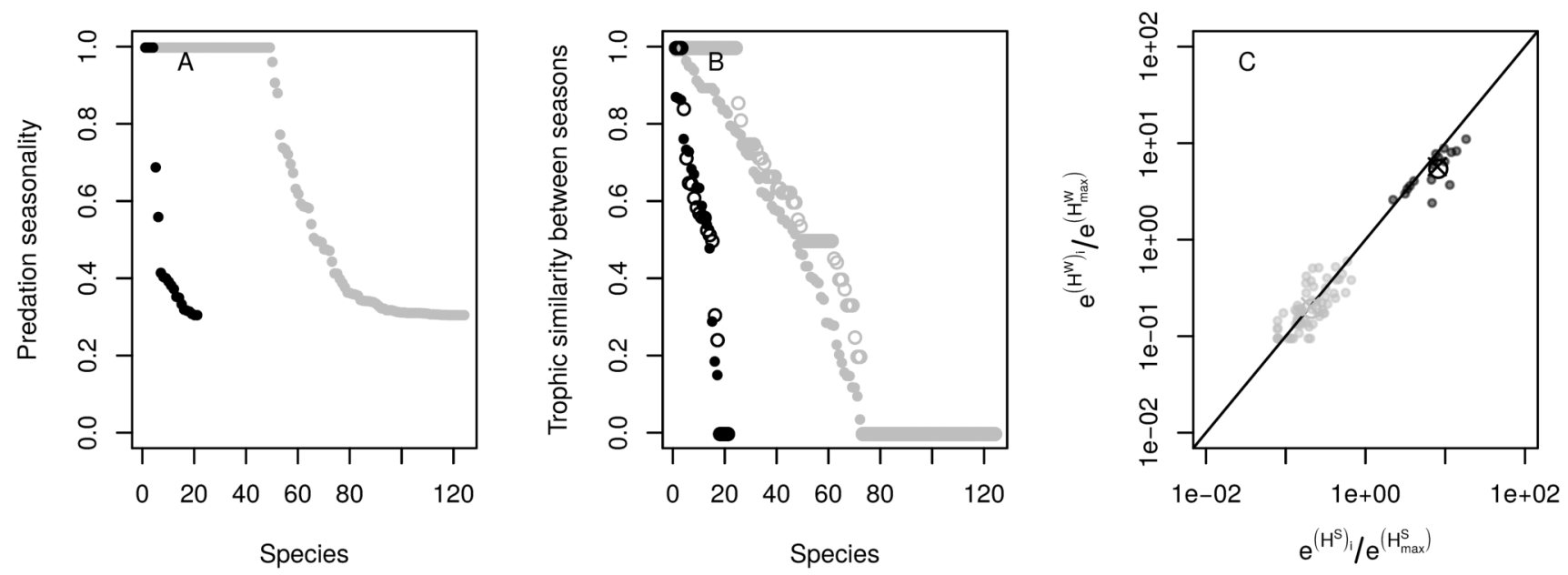

Figure 2: Seasonal changes of structure for the food web of the Białowieża forest. A) Predation seasonality for each species (from most seasonal to least seasonal). B) Trophic similarity between summer and winter for each species (species in decreasing order). Closed circles correspond to quantitative trophic similarity and open circles to qualitative trophic similarity. C) Winter proportional generality (vulnerability) against summer proportional generality (vulnerability) of each predator (prey) species $i$. Crossed circles represent the mean of each trophic level. For all graphs, each symbol corresponds to one species (black: predators, grey: prey).

\section{Parametrisation of a seasonally varying food web model}

\subsection{Basic bipartite predator-prey model}

To describe the dynamics of the predator-prey community, we use a general food web model, inspired by the Lotka-Volterra modelling of Saavedra et al. (2016). We modified predator growth rates by incorporating density-dependent mortality to account for the strong territoriality of species occurring in the Białowieża forest. The dynamics of predator and prey biomass densities $\left(C_{i}\right.$ and $R_{k}$ respectively) are described as follows for an arbitrary number of species:

$$
\left\{\begin{array}{c}
\frac{d C_{i}}{d t}=C_{i}\left(-m_{i}-g_{i} C_{i}+e \sum_{k} \frac{f_{k i}(\boldsymbol{R})}{M_{i}}\right) \\
\frac{d R_{k}}{d t}=R_{k}\left(r_{k}-\beta_{k} R_{k}\right)-\sum_{i} \frac{f_{k i}(\boldsymbol{R})}{M_{i}} C_{i}
\end{array}\right.
$$


where $m_{i}$ stands for the baseline mortality rate of predator $i, g_{i}$ for its density-dependent mortality rate (self-regulation), $e$ for the standard conversion efficiency, $r_{k}$ for the intrinsic growth rate of prey $k$, and $\beta_{k}$ for its intra-specific competition rate (set to 1 in Saavedra et al., 2016). The term $f_{k i}(\boldsymbol{R})$ is the per capita consumption rate of prey $k$ by predator $i$, i.e., its functional response (in g. $\left.\mathrm{y}^{-1} \cdot \mathrm{N}^{-1}\right)$. For the predation term of eq. (2) to have the adequate units in a biomass-based model, dividing the per capita functional responses of the predators by their body masses $M_{i}$ is required. This model formulation is aimed at keeping the parameters of predator functional responses in their original units to maximise reproducibility of the parameterisation process. In the present study, we consider both type I (eq. 3a) and type II (eq. 3b) multi-species functional responses which are respectively linear and saturating functions of prey densities:

$$
\begin{aligned}
& f_{k i}(\boldsymbol{R})=\gamma_{k i} R_{k} \\
& f_{k i}(\boldsymbol{R})=\frac{a_{k i} R_{k}}{1+h_{i} \sum_{j} a_{j i} R_{j}}
\end{aligned}
$$

where $\gamma_{k i}$ and $a_{k i}$ are the discovery rates of prey $k$ by predator $i$, and $h_{i}$ the handling time assuming it mostly depends on the predator physiological needs (Baudrot et al., 2016). Parameters definitions and units are summerised in Table 1. 


\begin{tabular}{|c|c|c|}
\hline Parameter & Definition & Unit \\
\hline$t$ & Time & $\mathrm{y}$ \\
\hline$C_{i}, R_{k}$ & Biomasses of predator $i$ and prey $k$ & g.ha ${ }^{-1}$ \\
\hline$r_{k}$ & Intrinsic growth rate of the prey $k$ & $\mathrm{y}^{-1}$ \\
\hline$\beta_{k}$ & Intra-specific competition rate of the prey $k$ & ha.g ${ }^{-1} \cdot \mathrm{y}^{-1}$ \\
\hline$m_{i}$ & Baseline mortality rate of the predator $i$ & $\mathrm{y}^{-1}$ \\
\hline$g_{i}$ & Density-dependent mortality rate of the predator $i$ & ha. $g^{-1} \cdot y^{-1}$ \\
\hline$e$ & Coefficient of conversion & - \\
\hline$M_{i}$ & Body mass of the predator $i$ & g. $\mathrm{N}^{-1}$ \\
\hline \multicolumn{3}{|c|}{ Type I functional response } \\
\hline$\gamma_{k i}(t)$ & Discovery rate & ha. $\mathrm{y}^{-1} \cdot \mathrm{N}^{-1}$ \\
\hline $\bar{\gamma}_{k i}$ & Average year-round discovery rate & ha. $\mathrm{y}^{-1} \cdot \mathrm{N}^{-1}$ \\
\hline$\left(\epsilon_{\gamma}\right)_{k i}$ & Magnitude of the discovery rate fluctuations & - \\
\hline \multicolumn{3}{|c|}{ Type II functional response } \\
\hline$a_{k i}(t)$ & Discovery rate & ha. $y^{-1} \cdot N^{-1}$ \\
\hline $\bar{a}_{k i}$ & Average year-round discovery rate & ha. $y^{-1} \cdot N^{-1}$ \\
\hline$\left(\epsilon_{a}\right)_{k i}$ & Magnitude of the discovery rate fluctuations & - \\
\hline $1 / h_{i}$ & Predator $i$ 's maximum intake rate & $g \cdot y^{-1} \cdot N^{-1}$ \\
\hline
\end{tabular}

Table 1: Parameters of the food web model. $\mathrm{N}^{-1}$ denotes per capita predator parameters.

\subsection{Parameters of predation}

We first estimate predation parameters defined in eq. 2 and eq. 3 for each season based on estimates of the per-capita intake of each prey population. Translation into time varying parameters is 
described in section 4 .

In the previous section, we defined $G_{k i}$, the biomass of prey $k$ harvested by an individual predator $i$ over an average year (in g. $\left.\mathrm{N}^{-1}\right)$. It is formally defined as:

$$
G_{k i}=\int_{y}^{y+1} f_{k i}(\boldsymbol{R}, t) d t
$$

where $f_{k i}(\boldsymbol{R}, t)$ is the instantaneous per capita consumption rate of prey $k$ by the predator $i$ in g. $\mathrm{y}^{-1} \cdot \mathrm{N}^{-1}$. In a seasonal food web model, not only prey densities vary throughout the year, but also the parameters of the functional response. In the present work, we specifically target the discovery rates $\gamma_{k i}$ and $\alpha_{k i}$, and estimate their values for each season assuming they are constant within each season. These two assumptions translate into functional responses which shift between two values, $f_{k i}^{S}\left(\boldsymbol{R}^{S}\right)$ and $f_{k i}^{W}\left(\boldsymbol{R}^{W}\right)$ for summer and winter respectively. $\boldsymbol{R}^{S}$ and $\boldsymbol{R}^{W}$ are the vectors of prey densities for summer and winter respectively. As the unit of these rates of intake is the gram per year per capita, eq. 4 simplifies to

$$
G_{k i}=G_{k i}^{S}+G_{k i}^{W} \simeq \eta^{S} f_{k i}^{S}\left(\boldsymbol{R}^{S}\right)+\eta^{W} f_{k i}^{W}\left(\boldsymbol{R}^{W}\right)
$$

when differentiating summer from winter intakes. $\eta^{S}$ and $\eta^{W}$ are the fractions of the year corresponding to summer and winter respectively $\left(\eta^{S}=0.46\right.$ and $\eta^{W}=0.54$ in the Białowieża forest). Assuming seasonal predator intakes to be governed by a linear functional response (i.e., type I as 
in eq. 3a), eq. 5 equates to

$$
\left\{\begin{array} { c } 
{ G _ { k i } ^ { S } = \eta ^ { S } \times \gamma _ { k i } ^ { S } \times R _ { k } ^ { S } } \\
{ G _ { k i } ^ { W } = \eta ^ { W } \times \gamma _ { k i } ^ { W } \times R _ { k } ^ { W } }
\end{array} \Rightarrow \left\{\begin{array}{l}
\gamma_{k i}^{S}=\frac{G_{k i}^{S}}{R_{k}^{S}} \times \frac{1}{\eta^{S}} \\
\gamma_{k i}^{W}=\frac{G_{k i}^{W}}{R_{k}^{W}} \times \frac{1}{\eta^{W}}
\end{array}\right.\right.
$$

where $R_{k}^{S}$ and $R_{k}^{W}$ are the biomass densities of the prey $k$ for summer and winter respectively. A type II functional response as described in eq. $3 \mathrm{~b}$ features a predator population split between two types of activities: $\left(C_{i}\right)_{s}$ are the predators searching for food, and $\left(C_{i}\right)_{h k}$ those handling prey $k$. Hence, we assume the main activity of a predator population is foraging (Koen-Alonso, 2007):

$$
C_{i}=\left(C_{i}\right)_{s}+\sum_{k}\left(C_{i}\right)_{h k}
$$

Herein, we use Baudrot et al. (2016)'s approach to describe the processes underlying predation governed by a type II functional response. $\alpha_{i}$ denotes the rate of prey accessibility for predator $i$, $p_{k i}$ the preference of predator $i$ for prey $k$, and $h_{k i}$ the handling time of prey $k$ by predator $i$ once killed. We hypothetise that the rate of prey accessibility $\alpha_{i}$ depends on the availability of predator $i$ 's prey so we write it as a function of their biomass densities $\boldsymbol{R}=\left\{R_{1}, R_{2}, \ldots\right\}$ :

$$
\alpha_{i}(\boldsymbol{R})=\sum_{j} a_{j i} R_{j}
$$

This rate corresponds to the total amount of prey encountered by each individual predator $i$ over the course of a year (in $\left.\mathrm{g} \cdot \mathrm{ha}^{-1} \cdot \mathrm{y}^{-1}\right)$. Similarly, we define the preference of predator $i$ for prey $k$ as a 
function of its relative biomass density:

$$
p_{k i}(\boldsymbol{R})=\frac{a_{k i} R_{k}}{\sum_{j} a_{j i} R_{j}}=\frac{a_{k i} R_{k}}{\alpha_{i}(\boldsymbol{R})}
$$

Hence, the functional response of predator $i$ to prey $k$ biomass density is

$$
f_{k i}(\boldsymbol{R})=\alpha_{i}(\boldsymbol{R}) \times p_{k i}(\boldsymbol{R}) \times \frac{\left(C_{i}\right)_{s}}{C_{i}}
$$

As predator reproduction happens at a slower rate than discovery and handling of prey, we assume the portion of newly born individuals contributing to the predation of a given prey species is marginal so that

$$
C_{i}=\left(C_{i}\right)_{s}\left(1+h_{i} \times \sum_{j} p_{i j}(\boldsymbol{R}) \times \alpha_{i}(\boldsymbol{R})\right)
$$

Whence,

$$
f_{k i}(\boldsymbol{R})=\frac{\alpha_{i}(\boldsymbol{R}) \times p_{k i}(\boldsymbol{R})}{1+h_{i} \times \alpha_{i}(\boldsymbol{R})}
$$

Replacing $p_{k i}(\boldsymbol{R})$ with eq. 9, we retrieve the formula for type II functional response in eq. 3b. Here, preferences $p_{k i}(\boldsymbol{R})$ are approximated with the biomass proportions $\omega_{k i}$ prey $k$ in predator $i$ 's diet, which are available for both predatory mammals' and raptors' diets (Jȩdrzejewska \& Jȩdrzejewski, 1998). The handling times $h_{i}$ can be estimated by considering the asymptotic value of $f_{k i}(\boldsymbol{R})$ when prey are non-limiting $\left(R_{k} \rightarrow \infty\right)$, in which case $f_{k i}(\boldsymbol{R}) \sim 1 / h_{i}$. We assume that an individual predator $i$ can harvest slightly beyond its own physiological needs (i.e., $(D F I)_{i} \times 365$ per year) by foraging as well for the juveniles of the year that it has to feed. Hence, the maximum intake of 
food biomass is estimated as

$$
1 / \hat{h_{i}}=\left[(D F I)_{i}+b_{i} \times(D F I)_{i}^{j u v}\right] \times 365 \Rightarrow \hat{h}_{i}=\frac{1}{\left[(D F I)_{i}+b_{i}(D F I)_{i}^{j u v}\right] \times 365}
$$

where $(D F I)_{i}^{j u v}$ is the daily food intake of juveniles of predator $i$ and $b_{i}$ the birth rate of the predator $i$ (see Table B2 in Supporting Information B for estimates).

With eq. 5 in mind, we write the intake of prey $k$ by predator $i$ during summer as

$$
G_{k i}^{S} \simeq \eta^{S} \times f_{k i}^{S}\left(\boldsymbol{R}^{S}\right)=\eta^{S} \times \frac{\alpha_{i}^{S}\left(\boldsymbol{R}^{S}\right) \times p_{k i}^{S}\left(\boldsymbol{R}^{S}\right)}{1+h_{i} \times \alpha_{i}^{S}\left(\boldsymbol{R}^{S}\right)}
$$

with a similar formula for winter intake $G_{k i}^{W}$. Based on these approximations, we first estimate the seasonal accessibility rates

$$
\begin{gathered}
\alpha_{i}^{S}\left(\boldsymbol{R}^{S}\right)=\frac{\sum_{k} 1 / \eta^{S} \times G_{k i}^{S}}{1-\sum_{k} 1 / \eta^{S} \times G_{k i}^{S} \times h_{i}} \\
\alpha_{i}^{W}\left(\boldsymbol{R}^{W}\right)=\frac{\sum_{k} 1 / \eta^{W} \times G_{k i}^{W}}{1-\sum_{k} 1 / \eta^{W} \times G_{k i}^{W} \times h_{i}}
\end{gathered}
$$

it follows for all $k$ and $i$

$$
\begin{aligned}
& a_{k i}^{S}=\frac{p_{k i}^{S}\left(\boldsymbol{R}^{S}\right) \times \alpha_{i}^{S}\left(\boldsymbol{R}^{S}\right)}{R_{k}^{S}} \\
& a_{k i}^{W}=\frac{p_{k i}^{W}\left(\boldsymbol{R}^{W}\right) \times \alpha_{i}^{W}\left(\boldsymbol{R}^{W}\right)}{R_{k}^{W}}
\end{aligned}
$$




\subsection{Intrinsic growth rates of the prey}

For prey intrinsic growth rates, we base our estimates on the central premise that they scale with species body mass following the quarter power law (Savage et al., 2004):

$$
r_{k} \propto M_{k}^{-1 / 4}
$$

where $M_{k}$ is the body mass of prey species $k$. To estimate $r_{k}$, we further assume that the relationship described by eq. 17 depends on the species group $g$ :

$$
r_{k, g}=E_{g} \times M_{k, g}^{-1 / 4}
$$

where $r_{k, g}$ and $M_{k, g}$ are the intrinsic growth rate and the body mass of species $k$ from group $g$. First, we calculate $E_{g}$ for various taxonomic groups thanks to the average body mass $\bar{M}_{g}$ (Supporting Information A) and the estimates of $\bar{r}_{g}$ we have for an average species of the group $g$ (Table B1 in Supporting Information B). We calculate $\bar{r}_{g}$ thanks to the estimated number $N_{\max }$ of juveniles produced by a pair of adults (Jȩdrzejewska \& Jȩdrzejewski, 1998), assuming an exponential growth

$\tilde{R}_{k}(t)=\tilde{R}_{0} \times e^{\hat{r}_{g} t}$ in an unbounded environment. Second, we estimate each $r_{k, g}$ based on eq. (18), where species body masses are picked from the literature (Fig. 3A). Missing values of intrinsic growth rates for other taxonomic groups are collected from the literature (Supporting Information B).

\subsection{Intra-specific competition rates of the prey}

In the absence of predators, our model described by eq. 2 assumes a logistic growth of prey species: at low densities, the prey growth follows their intrinsic growth rates and tapers off at higher densities 
because of intra-specific competition (each population reaches a plateau which corresponds to its carrying capacity). In model of eq. 2, the carrying capacity of a prey population $k$ is $\frac{r_{k}}{\beta_{k}}$ in the absence of predation. For all prey species, we infer the intra-specific competition rate $\beta_{k}$ as $r_{k} /\left(R_{k}\right)_{\max }$ (Fig. 3B), hence assuming that the highest biomass density recorded for a population matches its carrying capacity (see Supporting Information A for the estimation of prey carrying capacities).

\subsection{Mortality rates of the predators}

We estimate predator baseline mortality rates as the inverse of their maximum longevity $\Lambda_{i}$ (in years):

$$
m_{i}=1 / \Lambda_{i}
$$

We pick the greatest longevity values provided by the database AnAge (De Magalhães et al., 2005) to estimate baseline mortality rates. We verify that baseline mortality scaled with species body masses, by means of an analysis of covariance:

$$
\log \left(m_{i g}\right) \sim \bar{m}+\tau_{g}+\alpha \times\left(\log \left(M_{i, g}\right)-\log (\bar{M})\right)+\epsilon_{i g}
$$

where $m_{i g}$ is the baseline mortality of species $i$ in group $g, \bar{m}$ the mean mortality, $M_{i, g}$ the body mass of species $i$ of group $g$, and $\bar{M}$ the mean species body mass. We estimate $\tau_{g}$ the effect of group $g$ on the baseline mortality, the size $\alpha$ of the body mass effect, while $\epsilon_{i g}$ is the residual. Estimates of baseline mortality indeed scale with body mass, with coefficient $\alpha=-0.19$ (Fig. 3C). 


\subsection{Density-dependent mortality of the predators}

The term controlling the density-dependent mortality of the predators is key in regulating their densities and their impact on the prey community. To make our results robust to methodological choices regarding this parameter, we consider two alternative estimates of $g_{i}$ : a phenomenological estimate based on the observations and a model-based estimate.

The phenomenological estimate $\left(\hat{g}_{i}\right)_{\text {data }}$ relies on Jȩdrzejewska \& Jȩdrzejewski (1998)'s argument that year-round average values of predator biomass densities reflect fairly well how many individuals the Białowieża forest is able to support under pristine conditions. This equates to assuming constant predator densities, whence

$$
r_{i}-g_{i} \bar{C}_{i}=0 \Rightarrow\left(\hat{g}_{i}\right)_{d a t a}=\frac{\left(\hat{r}_{i}\right)_{o b s}}{\bar{C}_{i}}
$$

where $r_{i}=e \sum_{k} \frac{f_{k i}(\overline{\mathbf{R}})}{M_{i}}-m_{i}$ is the intrinsic growth rate of predator species $i$ (in $y^{-1}$, see eq. 2), $\left(\hat{r}_{i}\right)_{o b s}$ its estimate based on observational data, and $\bar{C}_{i}$ is the average year-round biomass density of predator $i$. We estimate $r_{i}$ with the observed ratio of biomasses during non-reproductive season to post-breeding season, assuming the breeding season lasts the whole summer (i.e., $\left(\hat{r}_{i}\right)_{o b s}=$ $\left.\frac{365}{168} \times \log \left(\frac{C_{i}^{\text {Autumn }}}{C_{i}^{\text {Spring }}}\right)\right)$.

The model-based estimate $\left(\hat{g}_{i}\right)_{\text {model }}$ uses the model structure, with the type II functional response which implies that predation is capped, to find out the regulation that has to be exerted to maintain a given predator density if predators ate at their maximum. This method uses as ingredients a theoretical predator maximum intrinsic growth rate $\left(\hat{r}_{i}\right)_{\max }$ and the maximum biomass density that the predator population could reach $\left(C_{i}\right)_{\max }$. We assume that $\left(\hat{r}_{i}\right)_{\max }$ is the intrinsic growth rate resulting from the maximum per capita intake rate $\left(\lim _{\mathbf{R} \rightarrow \infty} \sum_{k} f_{k i}(\mathbf{R})\right)$ and the baseline mortality 
$m_{i} .\left(C_{i}\right)_{\max }$ is arbitrarily set to $\left(\hat{C}_{i}\right)_{\max }=C_{i}^{A u t u m n} \times 1.5$ (e.g., juveniles of the year complete their growth). It ensues

$$
\left(\hat{g}_{i}\right)_{\text {model }}=\frac{\lim _{\mathbf{R} \rightarrow \infty} e^{\frac{\sum_{k} f_{k i}(\mathbf{R})}{M_{i}}}-m_{i}}{C_{i}^{\text {Autumn }} \times 1.5}
$$

where $\lim _{\mathbf{R} \rightarrow \infty} \sum_{k} f_{k i}(\mathbf{R})=\left[(D F I)_{i}+b_{i} \times(D F I)_{i}^{j u v}\right] \times 365=1 / h_{i}$ (see eq. 13). 

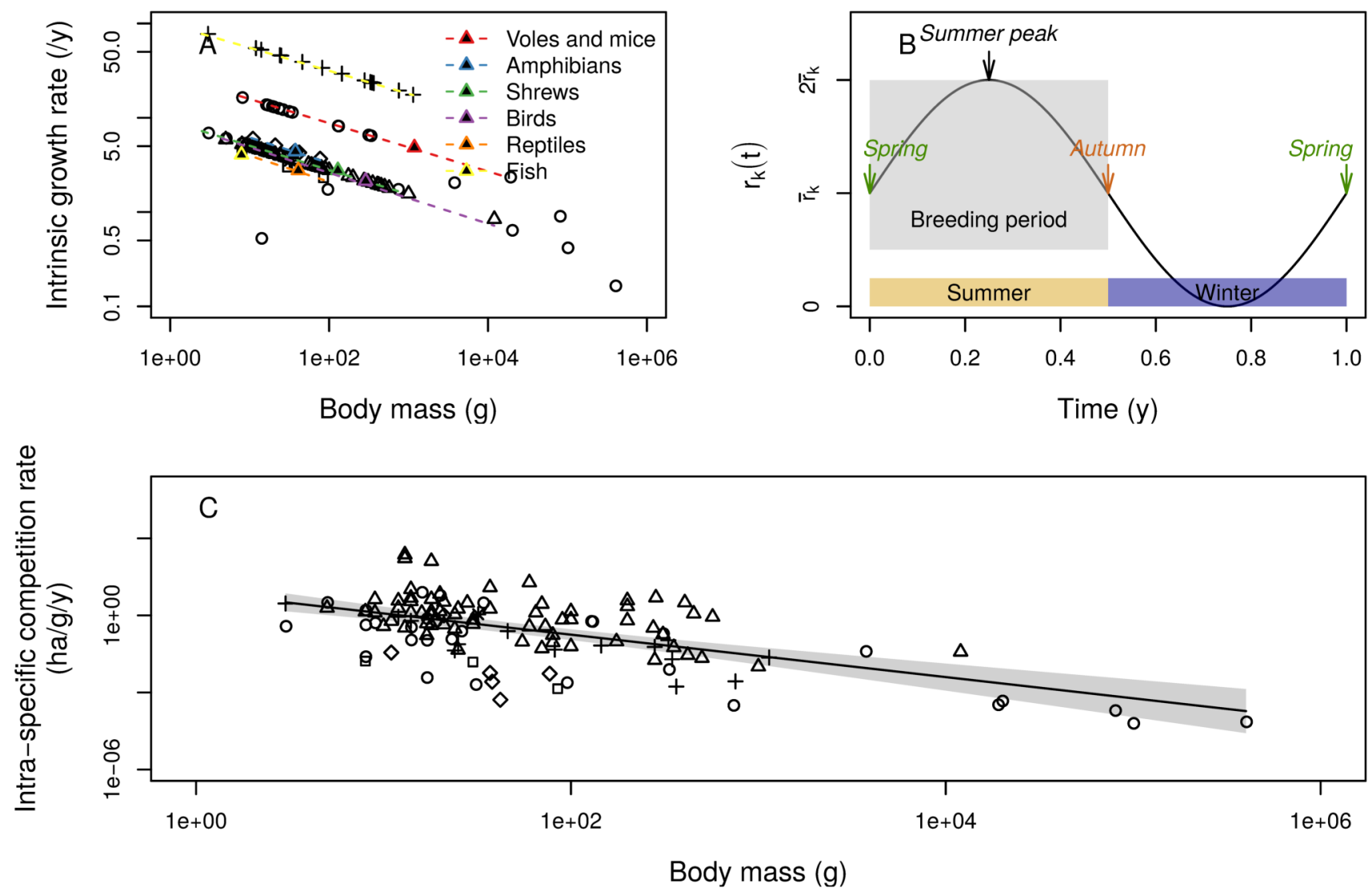

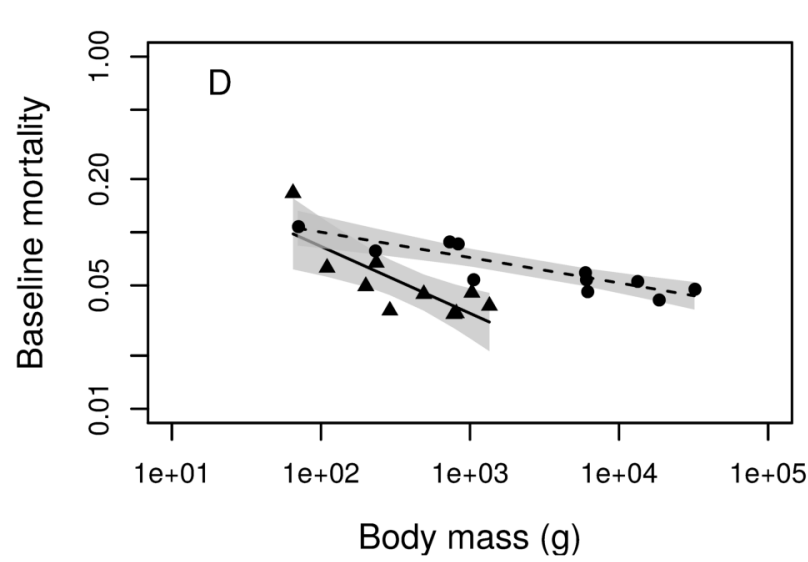

Trophic position

- Predator o Prey

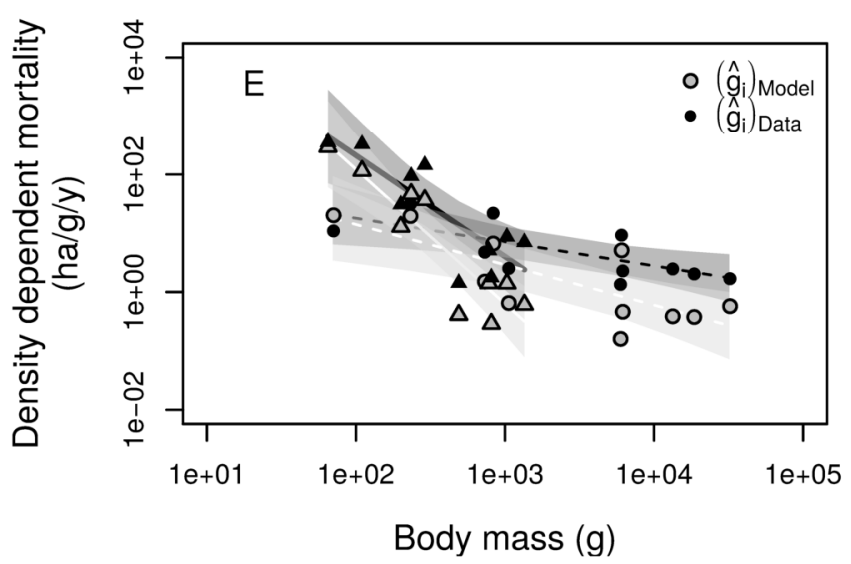

Species type

$\diamond$ Amphibian + Fish $\quad$ * Other

$\triangle$ Bird $\quad$ Mammal $\square$ Reptile

Figure 3: Parameters driving intra-specific growth with log-scaled axes, and their relationships with body mass.

(Figure caption continued on the following page.) 
Figure 3: (Continued figure caption.) A) Estimated intrinsic growth rates for prey species, where black upward triangles are reference points used to estimate the intercept of the line describing the relationship between $r_{k}$ and body masses for each group. Dashed lines indicate the relationship defined by eq. 18, with colours matching groups to which they apply. B) Phenology of prey reproduction as modelled in eq. 2 and eq. 23. C) Estimated intra-specific competition rates for prey species. D) Estimated baseline mortality rates for predator species and E) estimated density-dependent mortality rates for predator species either based on observed intrinsic growth rates (black symbols) or on maximum intakes of prey (grey symbols). In all plots, shaded areas cover the confidence intervals of the corresponding regression lines. Symbols correspond to species type.

\section{Simulating the seasonal food web of the Białowieża forest}

\subsection{Implementing seasonality}

The examination of the food web of the Białowieża forest highlights seasonal changes in the intakes of prey (Fig. 1) matching seasonal reproduction, changes in prey availability, or even presence of the predator in the study area (Jędrzejewska \& Jȩdrzejewski, 1998; Humphries et al., 2017). We implement predation seasonality by exerting a periodic forcing on the discovery rates - i.e., $\gamma_{k i}$ and $a_{k i}$, so they are now time-dependent.

In the previous section, for a given parameter $\chi_{k i}(t)$ shaping predation in our model, we actually estimate fixed rates $\chi_{k i}^{S}$ and $\chi_{k i}^{W}$ for summer and winter respectively. Following classic models of seasonal communities (Rinaldi et al., 1993), we assume that any time-varying parameter $\chi(t)$ can be decomposed into a mean value and a sinusoidal component:

$$
\chi(t)= \begin{cases}\bar{\chi}\left(1+\epsilon_{\chi} \times \sin (2 \pi t)\right) & \text { if highest during summer } \\ \bar{\chi}\left(1+\epsilon_{\chi} \times \sin (2 \pi t+\pi)\right) & \text { if highest during winter }\end{cases}
$$

where $\bar{\chi}$ is the average year-round parameter ( $\bar{\gamma}$ for discovery rates; $\bar{a}$ for attack rates), and $\epsilon_{\chi}$ is the relative magnitude of the parameter fluctuations (Table 1). This dimensionless parameter ranges 
from 0 - for constant discovery rates (i.e., $\chi(t)=\bar{\chi}$ for all $t$ ), to 1 - for interactions occurring mainly during a single season. Intermediate values of $\epsilon_{\chi}$ allow predation to fluctuate between seasons, and are intended to reflect changes in predatory behaviours over the course of the year.

To infer $\bar{\chi}_{k i}$ and $\left(\epsilon_{\chi}\right)_{k i}$, we assume $\chi_{k i}^{S}$ and $\chi_{k i}^{W}$ corresponds to the extremes values between which $\chi_{k i}(t)$ fluctuates over the course of the year. Given the periodicity of our forcing signal, mean rates are

$$
\bar{\chi}_{k i}=\frac{\chi_{k i}^{W}+\chi_{k i}^{S}}{2}
$$

and the forcing magnitude

$$
\left(\epsilon_{\chi}\right)_{k i}=\frac{\left|\chi_{k i}^{W}-\chi_{k i}^{S}\right|}{\chi_{k i}^{W}+\chi_{k i}^{S}}
$$

which simplifies to 1 if the predation link happens only during one season.

As seasonal reproduction is a pronounced feature of terrestrial temperate communities, we assume prey species reproduce mostly during summer. This is modelled by exerting a seasonal forcing on prey intrinsic-growth rates $r_{k}$ so it reaches its greatest value during summer (i.e., $r_{k}(t)=$ $\bar{r}_{k}(1+\sin (2 \pi t))$, where $\bar{r}_{k}=\hat{r}_{k}$, Fig. 3B).

\subsection{Modelling scavenging and predation on invertebrates}

In the Białowieża forest, predators may feed on a wide spectrum of resources, including plant material, invertebrates, and domestic animals. In the present model, we ignore most of these buffering resources to avoid unrealistic predator overpopulation, except when they constitute a substantial share of a predator's diet ( $>10 \%$ biomass). This includes earthworms for badgers 
(Meles meles), Hymenoptera for honey buzzards (Pernis apivorus), and carcasses for red foxes (Vulpes vulpes) and raccoon dogs (Nyctereutes procyonoides). The dynamics of these resources are likely to be very different from the rest of the community, as they operate at different time scales. Hereafter, we consider these food sources to be comparatively constant, so that the predator per capita intakes on these only vary with other prey species whose dynamics are effectively modelled by eq. 2 .

To ensure full reproducibility of the present model, we modify eq. 2 to include constant food sources. Hereafter, $\mathcal{A}_{i}$ denotes the set of constant feeding resources of predator $i$ so that $\sum_{k \in \mathcal{A}_{i}} \tilde{f}_{k i}(\boldsymbol{R}, t)$ is the instantaneous per-capita intake of constant feeding resources by the predator species $i$. The equation for predator dynamics now writes as

$$
\frac{d C_{i}}{d t}=C_{i}\left(-m_{i}-g_{i} C_{i}+e \sum_{k \notin \mathcal{A}_{i}} \frac{f_{k i}(\boldsymbol{R}, t)}{M_{i}}+e \sum_{k \in \mathcal{A}_{i}} \frac{\tilde{f}_{k i}(\boldsymbol{R}, t)}{M_{i}}\right)
$$

We now specify $\tilde{f}_{k i}(\boldsymbol{R}, t)$ so that eq. $3 \mathrm{a}$ and $3 \mathrm{~b}$ become, for constant food sources,

$$
\begin{aligned}
& \tilde{f}_{k i}(\boldsymbol{R}, t)=\tilde{\gamma}_{k i}(t) \\
& \tilde{f}_{k i}(\boldsymbol{R}, t)=\frac{\tilde{a}_{k i}(t)}{1+h_{i}\left(\sum_{j \notin \mathcal{A}_{i}} a_{j i}(t) R_{j}(t)+\sum_{j \in \mathcal{A}_{i}} \tilde{a}_{k i}(t)\right)}
\end{aligned}
$$

where $\tilde{\alpha}_{k i}(t)$ and $\tilde{\gamma}_{k i}(t)$ correspond to the amount of food resource $k$ that can be eaten per year by one predator $i$ (in g. $\mathrm{y}^{-1}$ ) for type I and type II functional responses. We then estimate the annual range for $\tilde{\alpha}_{k i}$ and $\tilde{\gamma}_{k i}$. For summer, the extrema are 


$$
\left\{\begin{array}{l}
\tilde{\gamma}_{k i}^{S}=\frac{G_{k i}^{s}}{\eta^{S}} \\
\tilde{\alpha}_{k i}^{S}=p_{k i}^{S}(\overline{\boldsymbol{R}}) \times \alpha_{i}^{S}(\overline{\boldsymbol{R}})
\end{array}\right.
$$

The formula are the same for winter but with different superscripts. Finally, the average values and the forcing magnitudes of $\tilde{\alpha}_{k i}(t)$ and $\tilde{\gamma}_{k i}(t)$ are calculated following eq. 24 and 25.

\subsection{Numerical integration}

We simulate the dynamics of the above-described food web model with our estimated parameter values. Simulations are initiated with the spring biomass densities reported from field studies, and run for 100 years. The system is numerically integrated with Matlab 2017b (The MathWorks Inc., Natick, Massachusetts, USA), using the function ode45 for numerical integration, with relative tolerance and absolute tolerance respectively set at $10^{-6}$ and $10^{-6}$. We set a biomass density threshold at $10^{-6}$ g.ha ${ }^{-1}$ (i.e., less than $1 \mathrm{~g}$ in the whole study area) below which a species is considered to be extinct.

All input parameters are given in Supporting Information C.

\section{Results from the simulations}

All simulations reach asymptotic dynamics within a few years of numerical integration (see the grey parts of the trajectories displayed in the phase portraits in Fig. 4). The simulations of our food web model for the Białowieża forest result in high level of species persistence (at least $78 \%$ of modelled species surviving), with all predators maintaining over the 100 years (Fig. 4). Greater predator density-dependent mortalities $\left(\hat{g}_{i}\right.$ based on observed growth during the breeding period, 
as in eq. 21) yields logically greater prey persistence. A predator intake dependent on prey density (type II functional response) decreases prey persistence (Fig. 4C-D), a somewhat surprising result, since predator intake is capped and in all other respects, the dynamics are similar to those with the type I response. As most extinctions occur in the early stages of the simulations (see black crosses on phase portraits of Fig. 4), these differences in persistence between variants of the model are not a mere result of the reduction of the speed at which species reach their extinction threshold.

All asymptotic dynamics are annual cycles. Both the type of functional responses and the method for estimating $g_{i}$ slightly modify the attractor shape (the set of biomasses densities produced by the simulations after transient dynamics), moving it to lower predator biomass densities and relatively smaller fluctuations for a type II response.

All the above results remain robust to varying initial conditions (Supporting Information D). We also found that marginal to moderate alterations of empirical abundance data ahead of the parameterisation had virtually no effect on community persistence (Supporting Information E). Yet, increasing or decreasing empirical abundance data ahead of the parameterisation of discovery rates can slightly modify the attractors (but not their overall shape and periodicities). Finally, model outcomes are more sensitive to the empirical abundance data of the most-studied groups (Fig. E3 and E4 in Supporting Information E), which may be simply the result of their larger contribution to trophic fluxes (Fig. E7). 

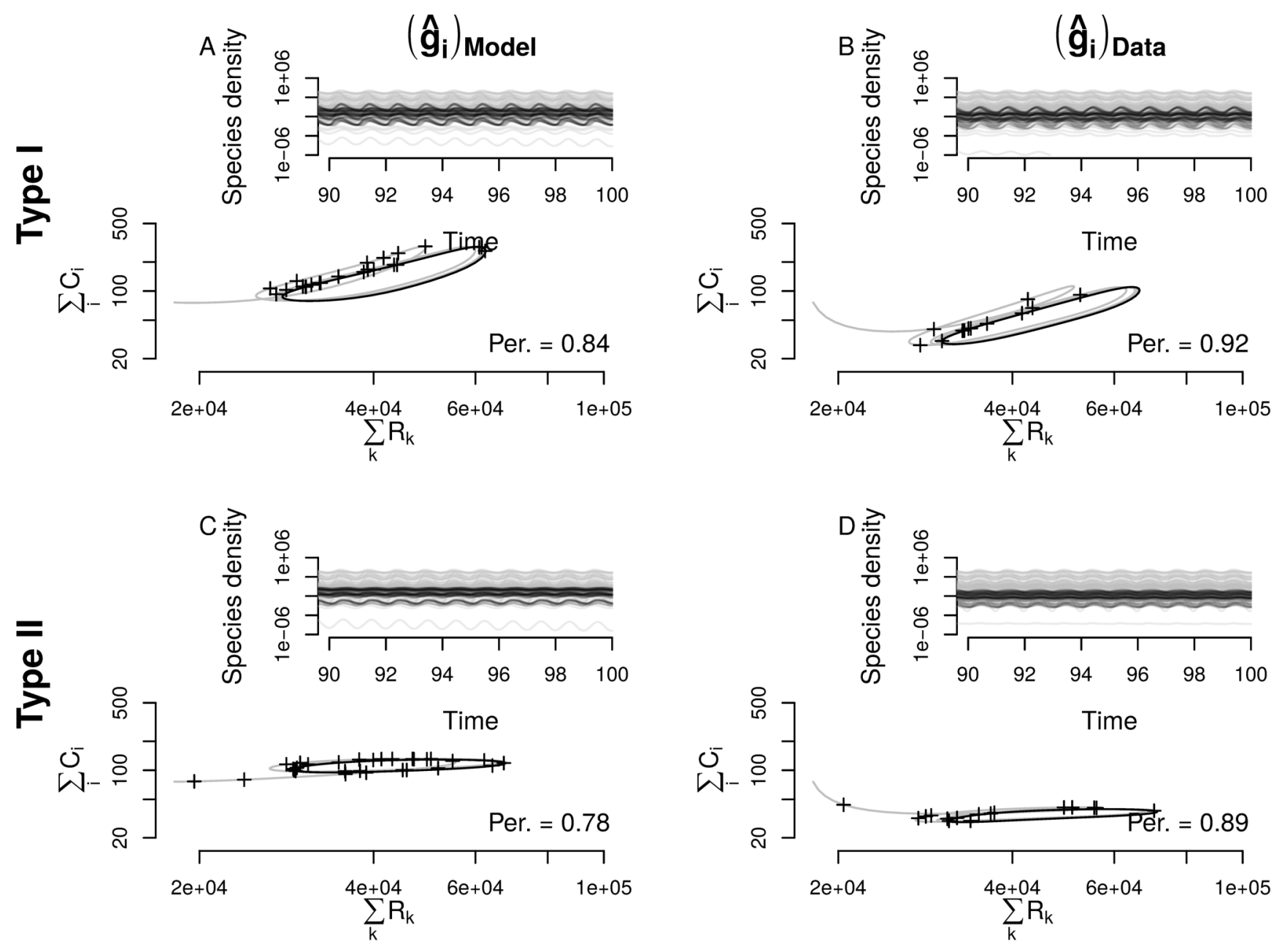

Figure 4: Simulated dynamics of the parameterised food web model for the forest of Białowieża displayed as phase portraits of densities aggregated by trophic level (in g.ha ${ }^{-1}$ ) and time series covering the last 10 years of simulation. Phase portraits represent the sum of predator densities $\sum_{i} C_{i}$ against the sum of prey densities $\sum_{k} R_{k}$, with transient dynamics in grey and asymptotic dynamics in black. Species extinctions are indicated with black crosses. In time series, each line corresponds to one species density over time, with prey taxa in grey and predators in black. For all simulations, the seasonal forcing is sinusoidal, and two types of functional responses are modelled: A-B) type I functional response; C-D) type II functional response. The predator density-dependent mortality is estimated either with eq. 22 (A and C), or with eq. 21 (B and D).

Our simulations reproduce observed densities at different seasons (spring, summer, autumn) with an error inferior to one order of magnitude for most species (and often much below, Fig. 5 and Supporting Information F). Exceptions are species for which we have little (reptiles and common mammals such as Lepus europaeus and Sciurus vulgaris) to no (fish community) empirical data 
on their seasonal biomasses. Note that errors are generally the largest for species with biomasses below 10 g.ha ${ }^{-1}$ : abundant species are almost all well-modelled.
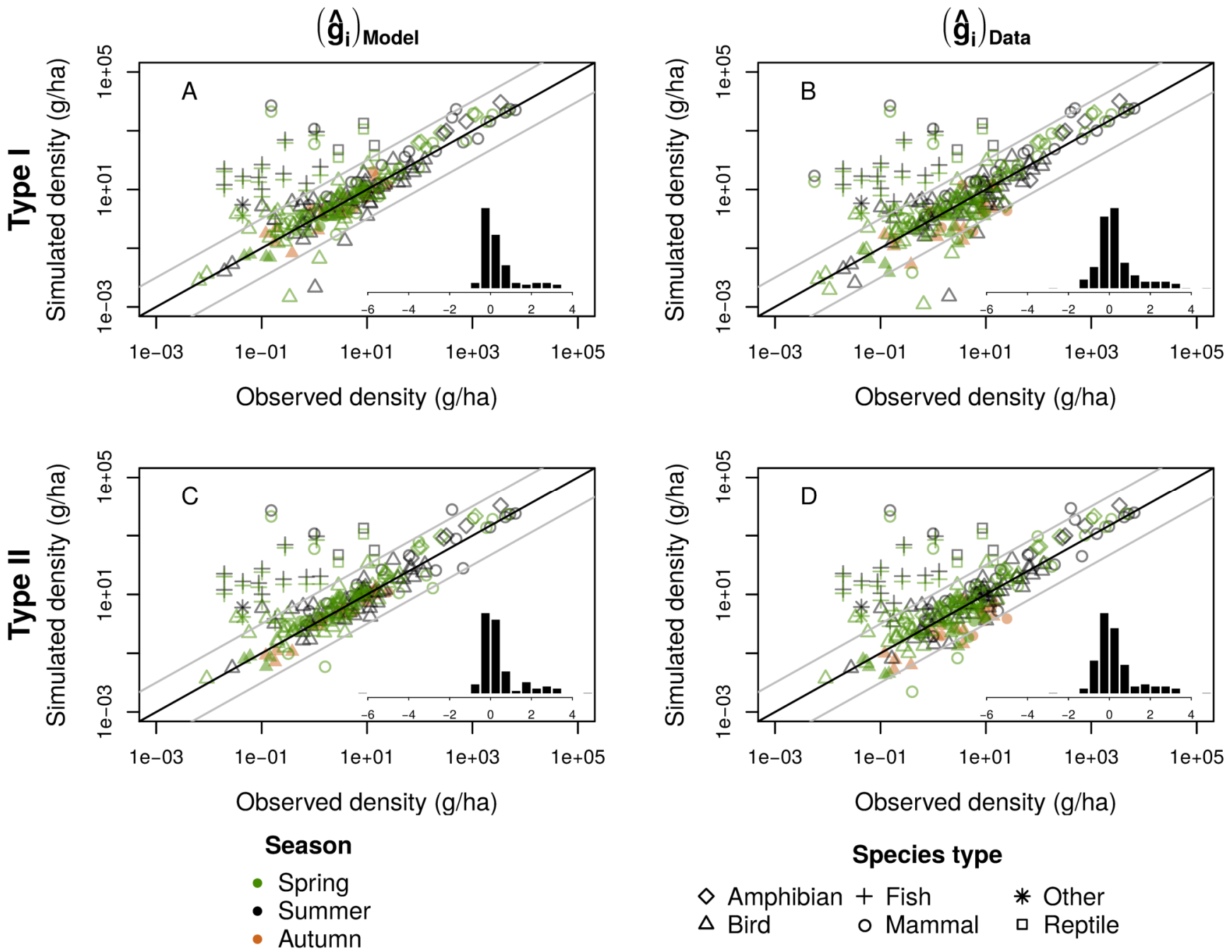

Figure 5: Comparison between observed $\left\{C_{i}^{\text {Obs }}, R_{k}^{\text {Obs }}\right\}$ and simulated densities $\left\{C_{i}^{\text {Sim }}, R_{k}^{\text {Sim }}\right\}$ for autumn (orange symbols, only for predators), summer (black symbols, only for prey species) and spring (green symbols). Note that axes are log-transformed. Comparison is drawn for type I (A-B) and type II (C-D) functional responses, as well as for two types of estimates for predator density-dependent mortality (A-C for $g_{i}$ according to eq. 22 and B-D when based on eq. 21). The grey lines frame species which estimated densities are similar to observations up to one order of magnitude. Insets represent the distribution of the simulated to observed density ratio $\left(\log _{10}\left(C_{i}^{\text {Sim }} / C_{i}^{\text {Obs }}\right)\right.$ for predator species, and $\log _{10}\left(R_{k}^{\text {Sim }} / R_{k}^{\text {Obs }}\right)$ for prey species), all seasons and species groups combined.

A close-up on comparisons of species abundances between seasons (spring versus autumn for predators; spring versus summer for prey) highlights that simulations preserve the overall trend to higher 
post-breeding biomasses (Fig. 6). Yet, seasonal variation of species biomasses can be strongly overestimated for some prey species (see symbols above the top grey line in Fig. 6C-D). But for most species at both trophic levels, the breeding season increase seems slightly underestimated. There are even a handful of cases where predator biomasses decrease slightly during the breeding season in the simulations (see symbols under the black line in Fig. 6A-B). In addition, the functional response and the estimating method of $g_{i}$ mostly affect predictions for predator seasonal growth by reducing their post-breeding biomasses (Fig. 6A-B). 

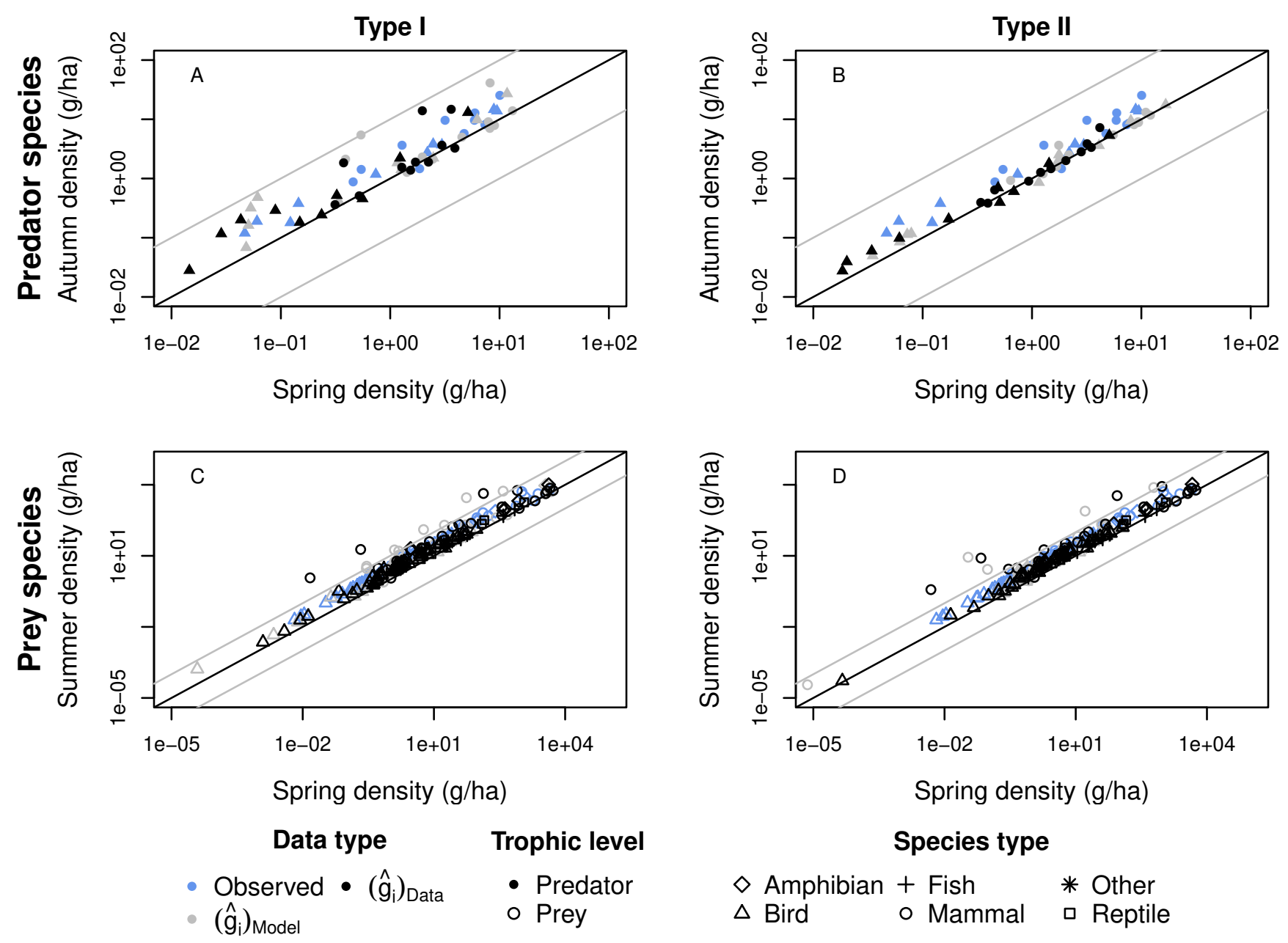

Figure 6: Seasonal variation of species densities. A-B) compare spring densities (when $t-\lfloor t\rfloor=0$ ) with autumn densities (when $t-\lfloor t\rfloor=0.5$ ) for predators while $\mathrm{C}-\mathrm{D}$ ) compare spring densities with summer densities (when $t-\lfloor t\rfloor=0.25$ ) for prey species. The identity of compared seasons is constrained by the data available to us. Observed biomass densities are plotted in blue, and simulated densities (seasonal densities averaged over 10 years) with grey $\left(\hat{g}_{i}\right.$ based on eq. 22$)$ and black symbols ( $\hat{g}_{i}$ based on eq. 21$)$. The black diagonals indicate equal densities between seasons. The grey lines frame seasonal variation within one order of magnitude.

We further quantify model fit with squared deviations to observed biomass values for each species (Fig. 7A-C). First, the estimation method for predator density-dependent mortality $\left(g_{i}\right)$ affects predator abundance less ambiguously than the abundance of prey species. Contrary to expectations, estimating $g_{i}$ with observed predator growth during the breeding season $\left(\left(g_{i}\right)_{\text {Data }}\right.$ in Fig. 7$)$ tends to decrease model accuracy. Second, differences in species squared deviations between methods 
for estimating predator density-dependent mortalities $g_{i}$ are more important when deviations are small (mostly for predator species). Meanwhile, when species biomasses are poorly predicted (high squared deviations), they are not improved by variants of the model or the estimating method of $g_{i}$. Thus, large deviations are mostly due to a lack of empirical knowledge and not a pitfall of either method for estimating $g_{i}$. Finally, shaping predation with a type II functional response does not improve the match between simulated and observed densities.

Overall, our parameterisation results in species abundance distributions (SADs) which are very similar to the observed SADs, displaying little variation in shape between seasons (Fig. 7B-D). This is especially true for predators (top lines in Fig. 7B-D) while SADs for prey species are slightly less skewed to lower densities and show truncated tails due to extinctions (bottom lines in Fig. 7B-D). Despite slight differences in species biomass predictions highlighted above, all model variants perform similarly in terms of SAD. 

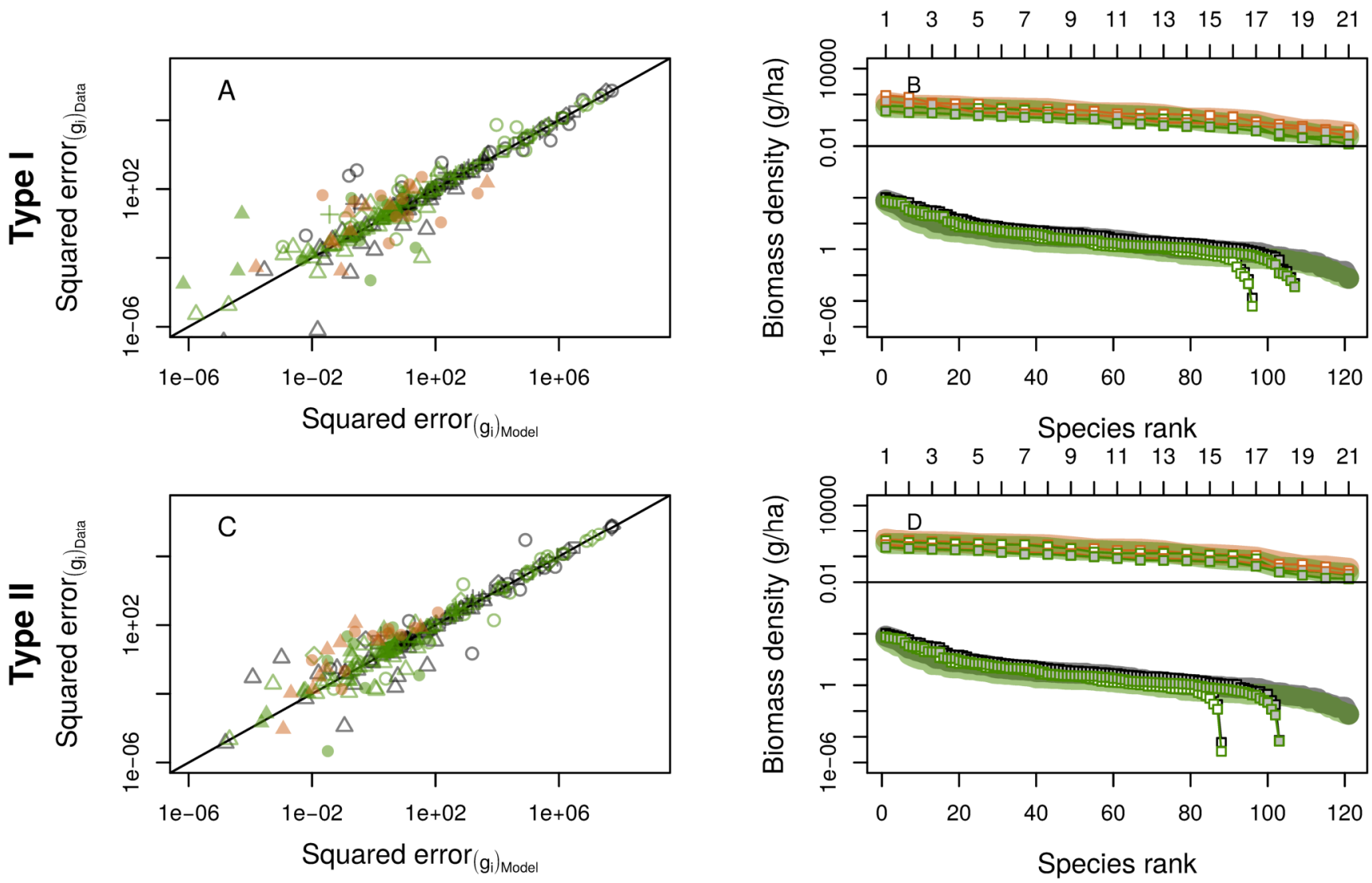

Season

Species type

- Spring

- Summer

$\diamond$ Amphibian o Mammal

- Autumn
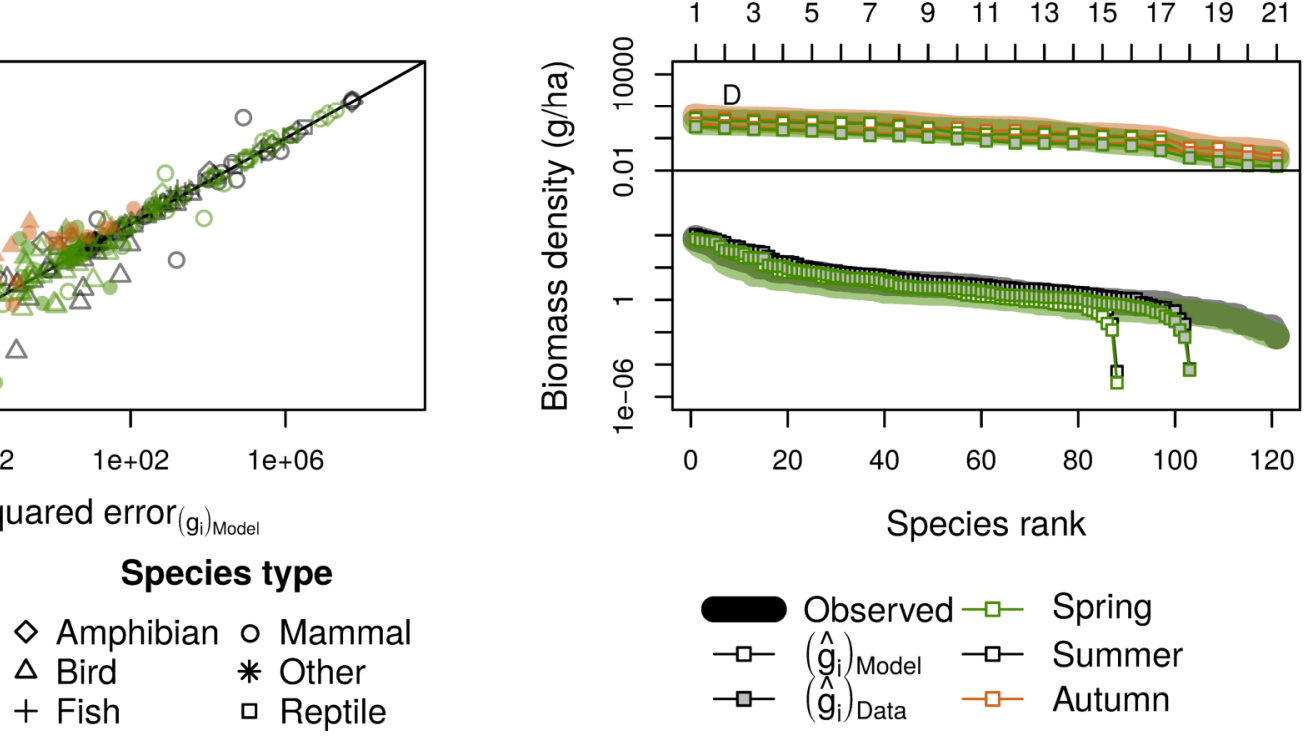

Figure 7: Comparisons of model-predicted biomasses to observed data in terms of A-C) species squared errors (solid symbols for predators and open symbols for prey) and B-D) species abundance distributions for predators (top) and prey species (bottom). Model quality is investigated for both type I (A-B) and type II (C-D) functional responses, as well as for two types of $g_{i}$ estimates. Green symbols correspond to spring densities, black symbols to summer densities, and orange symbols to fall densities.

\section{Discussion}

Building on the extensive empirical studies conducted in the Białowieża forest, summarised by Jȩdrzejewska \& Jȩdrzejewski (1998), we have parameterised a dynamic seasonal food web model with type I and type II functional responses. Our methodology combines information about seasonal 
diets, physiological needs of the predators, life-history characteristics of both prey and predator species, and observed seasonal fluctuations of densities. The corresponding simulations yielded at least about $80 \%$ persistence of the modelled species, a good score given the entirely additive effects of predation. We also had a good quantitative match to observed biomasses, for the most part much below one order of magnitude, despite some seasonal variation in accuracy. Yet, there seems to be a trade-off between improving species persistence and improving the quantitative match to the observed biomasses. We explore these results and their implications in more detail below; we start with technical considerations regarding this parameterisation exercise before moving to more ecological considerations aimed at improving seasonal food web modelling more generally.

\subsection{Trade-off between improving species persistence and model accuracy}

In our attempt to parameterise the seasonal food web of the Białowieża forest, we considered two methods for estimating the predator density-dependent mortality coefficient $g_{i}$. One was based on the theoretical maximum intakes and densities of the predators, and the other based on observed growth rates and densities. We also considered two variants of the predators functional response (Holling types I and II). Both choices affect predator intrinsic growth and their impact on prey populations. Therefore, we expected that model variants could have very different persistence levels and model accuracy (i.e., distance between observed and predicted biomasses).

Estimates of predator density-dependent mortality rates based on observed growth rates resulted in greater persistence but also larger differences between simulated and observed seasonal densities, at both trophic levels. In other words, model accuracy for persisting species traded off with species

persistence. Greater persistence is easily explained as this method produces higher estimates of predator density-dependent mortality rates (Fig. 3E) indirectly decreasing predatory pressure on 
prey populations through reduced predator populations. Yet, although using observational data for estimating $g_{i}$ could be thought to produce more life-like biomass densities (and perhaps some overfitting), it actually increased squared deviations for predators. We further discuss predator regulation in sections 6.3 (competition for territories) and 6.4 (intra-guild predation).

A saturating functional response caps predator foraging capacity, usually helping prey populations to maintain at higher densities. We dismissed S-shaped functional responses because of potential artefacts induced by their use for large food webs (Morozov \& Petrovskii, 2013) to opt for a simpler type II functional response (consistent with Jȩdrzejewska \& Jȩdrzejewski, 1998). Greater model accuracy was expected with the type II functional response, while overestimated consumption for abundant prey species was likely with the type I response. Rather, simulations produced lower persistence for the prey community while simulated species biomasses were not uniformly improved. One possible lead to explain these results lies in the parameterisation of the discovery rates: under the assumption of a type II functional response, estimated discovery rates for a given consumption rate are larger. Another possibility is that the cap on predator foraging rates introduce slight delays in predator population build-up, which eventually causes prey to suffer more predation later on, and persistence to decrease overall. A sensitivity analysis examining to what extent the model dynamical outcomes can be modified in response to changes in empirical abundance data (see eq. 6 and 16) highlights that the type II functional response decreases model sensitivity to abundance data. Hence, although the type II functional response may seem to fit a little less well to observational data, describing predation with a saturating functional response limits the potentially detrimental effect of sampling errors and uncertainties on food web parameterisation (Supporting Information E). However, one should keep in mind that the differences between our model variants are relatively small and should not be overinterpreted. 


\subsection{Mimicking annual cycles}

Our parameterised food web model generates annual cycles, throughout which the model reproduces fairly well spring biomasses but a little less well summer and fall biomasses. In other words, despite the seasonal forcing that we implemented, our model does not fully account for the processes occurring during the breeding season. Because of the energy requirements for reproduction, breeding often coincides with greater food availability (Jönsson et al., 1998). Following Rinaldi et al. (1993), we have thus considered a prey intrinsic growth rate that fluctuates seasonally while assuming that intra-specific competition remains constant. Yet, it is possible that several prey parameters fluctuate seasonally. Indeed, plant growth and fruiting, as well as insect emergence occur periodically following temperature trends, and affect directly the food stocks available to herbivores and insectivores (Humphries et al., 2017). Eventually, greater resource stocks for prey could correspond not only to an increased maximal growth but also a greater prey carrying capacity in summer, both of which could combine to massively decrease the intra-specific competition in summer. Seasonally fluctuating prey competition is therefore one possible improvement for this model framework.

Another source of seasonal fluctuation in the empirical version of this food web relative to its theoretical idealisation is migration: many prey species of this food web migrate (mostly insectivorous birds). In the present work, we take into account the absence of these birds with a seasonal forcing on predation so that it is interrupted in winter. Yet, springtime immigration provides a great increase in overall biomass in the forest (Jȩdrzejewska \& Jȩdrzejewski, 1998). If the predator has a strong numerical response, sudden prey availability is expected to boost predation at the expense of resident prey sharing the same predator (e.g. wood warblers competiting with rodents; Grendelmeier et al., 2018), and to magnify this apparent competition which already occurs in our model. 
However, modelling the actual biomass upsurge in the spring could also enable apparent mutualism, provided that predators respond to prey availability by switching or have weak numerical response (Holt \& Bonsall, 2017). It is thus difficult to predict how implementing a more realistic seasonal migration would affect the dynamics of the community.

\subsection{Multi-annual dynamics}

Many species of the modelled food web are known to display multi-annual dynamics (commonly 3 to 5-year cycles), with shrews and voles being the most well-known cases (Pucek et al., 1993; Stenseth et al., 2002). Models such as ours are susceptible to produce limit cycles or chaotic attractors when unforced (Bazykin, 1998) and periodic forcing can create or enhance multi-annual cycles (e.g., Rinaldi et al., 1993; Taylor et al., 2013). Although the "right ingredients" for multiannual cycles in predator-prey systems are gathered in our parameterised model (high prey growth rates, saturating functional responses in the type II case, periodic forcing for prey growth), none of the modelled species had multiannual dynamics in our simulations. Therefore, the predation hypothesis, whereby some strongly cyclic species oscillate because of predators, and also drag many other species with them through shared predation (e.g., Kjellander \& Nordström, 2003; Korpimäki et al., 2005), could seem unlikely on its own.

Although predation may not explain the observed multi-annual dynamics of some species like rodents, a contributing factor to the relative stability of the community dynamics may be our choice of Bazykin's model for predator self-regulation, that is, a quadratic mortality term (Bazykin, 1998). With our empirically-based parameterisation with strong predator self-regulation, limit

cycles would be rare in the absence of seasonal forcing (Metzler \& Wischniewsky, 1985). It is therefore logical that multi-annual cycles do not appear here: even with the seasonal forcing exciting 
the dynamical system, there is too much dampening of the predator growth rate. Alternative formulations of self-regulation that model territory size as inversely proportional to prey density can produce multi-annual cycles when seasonally forced (Turchin \& Hanski, 1997), but whether they do so more often than the Bazykin form depends on parameter ranges (Turchin \& Batzli, 2001). It is also unclear how to formulate them for multiple species of predators and prey. Hence, while the modelling of predator territoriality could be improved somewhat, Bazykin's formulation provides a suitable first approximation. Self-regulation of predators, through territoriality or other means, is quite likely both generating persistence (as in other food webs, Barabás et al., 2017) and dampening fluctuations in this system.

An alternative - though not mutually exclusive - explanation for the absence of multi-annual cycles in this system may be that they are largely driven by mechanisms that are not currently implemented in the models. Other sources of forcing, at the multi-annual rather than seasonal scale, also prevail in the forest. For instance, some prey intrinsic growth rates are themselves fluctuating over several years, due to fluctuations in resources such as seeds (e.g, McShea, 2000) due to tree masting (oak, hornbeam, maple, and spruce), or Lepidoptera (e.g., Ludwik \& Wesołowski, 1996). It may provide yet another "push" for the food web model to start oscillating. However, it is possible that the high level of predator regulation in our model actually precludes such multiannual oscillations, even with more temporal forcing. Moreover, stage-structure, which we entirely neglected here, is known to likely cause the majority of short-period population cycles (Murdoch et al., 2002), possibly in interaction with predation. It is therefore another potential direction for model improvement. 


\subsection{Food web structure}

Two additional simplifying features of our model, that pertain to the structure of the network, are that: (1) it considers a bipartite web and (2) it assumes well-mixed populations in a homogeneous environment. Given these two simplifying assumptions, and the ones mentioned in the previous section, we find remarkable that the model manages to mimic most of the essential features of this forest food web. But relaxing these assumptions may lead to better prediction, especially for the species that are currently less well modelled (either too abundant or extinct in our simulations). The main implication of a bipartite predator-prey web is to forbid omnivory within a guild while this is widespread at upper trophic levels (Thompson et al., 2007). According to Jȩdrzejewska \& Jȩdrzejewski (1998), intraguild predation in the Białowieża forest may occur through actual hunting of smaller taxa by larger ones such as raptors preying on mustelids (e.g., Korpimäki \& Norrdahl, 1989; Zub et al., 2008) or mustelids consuming raptors' eggs or juveniles. Omnivory also includes interference competition by which dominant predators kill their competitors (e.g., lynxes killing foxes, Sunde et al., 1999), and possibly consume them. Yet, it is difficult to identify whether empirically found intra-guild links correspond to actual predation and affect the victim's population when using data based on scats and pellets: overestimating omnivory is possible as other predators' presence in another diet may be simply the trace of scavenging behaviour (Jȩdrzejewska \& Jȩdrzejewski, 1998). Still, a more accurate modelling of omnivory could enable a more realistic predation regulation and possibly alleviate limitations of our model (Rudolf, 2007a,b).

Another important simplification pertains to space and the arrangements of habitats. Although most of the Białowieża forest is obviously covered by woodland, this study site comprises very diverse forest habitats (e.g., oak-lime-hornbeam forest, mixed spruce-oak-pine forest, pure coniferous) as well as open marshes, clearings, artificial lakes and rivers. The latter habitats host distinct 
prey populations which locally thrive while they are rarer elsewhere in the Białowieża forest (e.g., hares, some microtine species, marsh birds, fish). Interestingly, these species are actually those that we fail to model, as they go extinct while they have life-history parameters that usually allow for clear persistence in their preferred habitats. Indeed, assuming well-mixed populations in homogeneous habitats forces to consider population densities averaged over the whole study area, which underestimated prey carrying capacities (overestimated prey competition rates) for these open-habitat species. This makes them more extinction-prone that they actually are. In addition, their predators may either occupy forest ecotones, splitting their foraging effort between the prey communities occupying different habitats (e.g., long-eared owls and red foxes). Ignoring this spatial distribution of predator-prey interactions likely overestimates discovery rates of these prey species as their densities are low at the scale of the whole Białowieża forest but not at the scale of their predator's hunting territories. Therefore, a spatially differentiated model (i.e., with several habitats) may allow greater persistence still for these open-habitat prey species.

\subsection{Conclusion and perspectives}

Using a richly studied predator-prey vertebrate community, the Białowieża forest food web, we parameterised a fully dynamic seasonal food web model. Numerical integration of this model allowed to reproduce the observed biomasses, their seasonal changes, and the species abundance distributions. The model predicted dynamics of annual periodicity for the whole community, which mimicks the seasonal rythms observed in the forest. Although some aspects of model structure can be improved in future work (territoriality, migration, spatial structure, omnivory), we showed that it is possible to use seasonal descriptions of diet and biomasses, combined with life-history data, to parameterise a differential equations model with time-varying parameters. In that sense, we 
extend the work on seasonal predator-prey modules (e.g., Turchin \& Hanski, 1997; Gilg et al., 2003) to the food web scale. A similar methodology may be applied to other well-studied ecosystems ranging from boreal forests (e.g., the Kluane National Park and Reserve; Krebs et al., 2001) to African savannas (e.g., the Serengeti National Park; Sinclair \& Arcese, 1995) where the specifics of seasonality may vary.

Overall, as food web studies are moving beyond a frozen picture of ecological communities (e.g., Saavedra et al., 2016; Ushio et al., 2018), parameterised seasonal food-web models enable in silico tests of the contribution of environmental fluctuations to community structure (e.g., Mellard et al., 2019) and dynamics. In the long run, modelling seasonal food webs should allow to better understand how alterations of seasonal rythms may affect long-term dynamics and community structure.

\section{Acknowledgements}

This work was funded by the French ANR through LabEx COTE (ANR-10-LABX-45). We are very much indebted to all the researchers who performed the studies of trophic interactions in the Białowieża forest, on which our model is based, especially B. Jẹdrzejewska and W. Jȩdrzejewski who gathered all of this knowledge in a comprehensive book, without which the present work would have not been possible. We also thank two anonymous reviewers for their constructive comments to improve this paper. 


\section{Authors' contributions}

AMCS and FB conceived the project, designed the mathematical models, as well as how to estimate model parameters; AMCS digitised and compiled all the data, translated the methodology into code, performed network analyses and ran the dynamic model simulations; AMCS and FB then jointly decided on the manuscript structure. The writing was led by AMCS with inputs from FB.

\section{Data availability statement}

Data and computer codes for analyses are available in GitHub repository alixsauve/SeasonalFoodWeb and have been archived at Zenodo with https://doi.org/10.5281/zenodo.3720260.

\section{References}

Barabás, G., Michalska-Smith, M.J. \& Allesina, S. (2017) Self-regulation and the stability of large ecological networks. Nature ecology \& evolution 1, 1870-1875.

Baudrot, V., Perasso, A., Fritsch, C., Giraudoux, P. \& Raoul, F. (2016) The adaptation of generalist predators' diet in a multi-prey context: insights from new functional responses. Ecology $\mathbf{9 7}, 1832-$ 1841.

Bazykin, A.D. (1998) Nonlinear dynamics of interacting populations. World Scientific.

Boit, A., Martinez, N.D., Williams, R.J. \& Gaedke, U. (2012) Mechanistic theory and modelling of complex food-web dynamics in lake constance. Ecology letters 15, 594-602.

Brose, U., Archambault, P., Barnes, A.D., Bersier, L.F., Boy, T., Canning-Clode, J., Conti, E., 
Dias, M., Digel, C., Dissanayake, A. et al. (2019) Predator traits determine food-web architecture across ecosystems. Nature Ecology \& Evolution 3, 919.

Brose, U., Williams, R.J. \& Martinez, N.D. (2006) Allometric scaling enhances stability in complex food webs. Ecology letters 9, 1228-1236.

Chevillot, X., Tecchio, S., Chaalali, A., Lassalle, G., Selleslagh, J., Castelnaud, G., David, V., Bachelet, G., Niquil, N., Sautour, B. et al. (2019) Global changes jeopardize the trophic carrying capacity and functioning of estuarine ecosystems. Ecosystems 22, 473-495.

Christensen, V. \& Pauly, D. (1992) Ecopath II - a software for balancing steady-state ecosystem models and calculating network characteristics. Ecological modelling 61, 169-185.

Curtsdotter, A., Banks, H.T., Banks, J.E., Jonsson, M., Jonsson, T., Laubmeier, A.N., Traugott, M. \& Bommarco, R. (2019) Ecosystem function in predator-prey food webs - confronting dynamic models with empirical data. Journal of Animal Ecology 88, 196-210.

De Magalhães, J.P., Costa, J. \& Toussaint, O. (2005) HAGR: the human ageing genomic resources. Nucleic Acids Research 33, D537-D543.

de Ruiter, P.C., Neutel, A.M. \& Moore, J.C. (1995) Energetics, patterns of interaction strengths, and stability in real ecosystems. Science 269, 1257-1260.

DeLong, J.P., Gilbert, B., Shurin, J.B., Savage, V.M., Barton, B.T., Clements, C.F., Dell, A.I., Greig, H.S., Harley, C.D., Kratina, P. et al. (2015) The body size dependence of trophic cascades. The American Naturalist 185, 354-366.

Dormann, C.F., Fründ, J., Blüthgen, N. \& Gruber, B. (2009) Indices, graphs and null models: analyzing bipartite ecological networks. The Open Ecology Journal 2, 7-24. 
Gauzens, B., Barnes, A., Giling, D.P., Hines, J., Jochum, M., Lefcheck, J.S., Rosenbaum, B., Wang, S. \& Brose, U. (2019) fluxweb: An R package to easily estimate energy fluxes in food webs. Methods in Ecology and Evolution 10, 270-279.

Gilg, O., Hanski, I. \& Sittler, B. (2003) Cyclic dynamics in a simple vertebrate predator-prey community. Science 302, 866-868.

Grendelmeier, A., Arlettaz, R. \& Pasinelli, G. (2018) Numerical response of mammalian carnivores to rodents affects bird reproduction in temperate forests: A case of apparent competition? Ecology and Evolution 8, 11596-11608.

Hanski, I. \& Korpimäki, E. (1995) Microtine rodent dynamics in northern europe: parameterized models for the predator-prey interaction. Ecology 76, 840-850.

Holt, R.D. \& Bonsall, M.B. (2017) Apparent competition. Annual Review of Ecology, Evolution, and Systematics 48, 447-471.

Hudson, L.N. \& Reuman, D.C. (2013) A cure for the plague of parameters: constraining models of complex population dynamics with allometries. Proceedings of the Royal Society B: Biological Sciences 280, 20131901.

Humphries, M.M., Studd, E.K., Menzies, A.K. \& Boutin, S. (2017) To everything there is a season: summer-to-winter food webs and the functional traits of keystone species. Integrative and Comparative Biology 57, 961-976.

Jȩdrzejewska, B. \& Jędrzejewski, W. (1998) Predation in vertebrate communities: the Biatowieża Primeval Forest as a case study. Springer Science \& Business Media. 
Jönsson, K.I., Tuomi, J. \& Järemo, J. (1998) Pre- and postbreeding costs of parental investment. Oikos 83, 424-431.

Kalinkat, G., Rall, B.C., Vucic-Pestic, O. \& Brose, U. (2011) The allometry of prey preferences. PloS one 6, e25937.

Kartascheff, B., Heckmann, L., Drossel, B. \& Guill, C. (2010) Why allometric scaling enhances stability in food web models. Theoretical Ecology 3, 195-208.

Kjellander, P. \& Nordström, J. (2003) Cyclic voles, prey switching in red fox, and roe deer dynamics-a test of the alternative prey hypothesis. Oikos 101, 338-344.

Koen-Alonso, M. (2007) A process-oriented approach to the multispecies functional response. From energetics to ecosystems: the dynamics and structure of ecological systems, pp. 1-36, Springer.

Korpimäki, E. \& Norrdahl, K. (1989) Avian predation on mustelids in Europe 1: occurrence and effects on body size variation and life traits. Oikos 55, 205-215.

Korpimäki, E., Norrdahl, K., Huitu, O. \& Klemola, T. (2005) Predator-induced synchrony in population oscillations of coexisting small mammal species. Proceedings of the Royal Society B: Biological Sciences 272, 193-202.

Krebs, C.J., Boutin, S. \& Boonstra, R. (eds.) (2001) Ecosystem dynamics of the boreal forest: the Kluane project. Oxford University Press.

Legagneux, P., Gauthier, G., Berteaux, D., Bêty, J., Cadieux, M.C., Bilodeau, F., Bolduc, E., McKinnon, L., Tarroux, A., Therrien, J.F. et al. (2012) Disentangling trophic relationships in a High Arctic tundra ecosystem through food web modeling. Ecology 93, 1707-1716. 
Ludwik, T. \& Wesołowski, T. (1996) Structure of a primeval forest bird community during 1970s and 1990s (Białowieża National Park, Poland). Acta Ornithologica 31.

McShea, W.J. (2000) The influence of acorn crops on annual variation in rodent and bird populations. Ecology 81, 228-238.

Mellard, J.P., Audoye, P. \& Loreau, M. (2019) Seasonal patterns in species diversity across biomes. Ecology 100, e02627.

Metzler, W. \& Wischniewsky, W. (1985) Bifurcations of equilibria in Bazykin's predator-prey model. Mathematical Modelling 6, 111-123.

Morozov, A. \& Petrovskii, S. (2013) Feeding on multiple sources: towards a universal parameterization of the functional response of a generalist predator allowing for switching. PloS one 8 , e74586.

Murdoch, W.W., Kendall, B., Nisbet, R., Briggs, C., McCauley, E. \& Bolser, R. (2002) Singlespecies models for many-species food webs. Nature $\mathbf{4 1 7}, 541$.

Pucek, Z., Jędrzejewski, W., Jędrzejewska, B. \& Pucek, M. (1993) Rodent population dynamics in a primeval deciduous forest (Białowieża National Park) in relation to weather, seed crop, and predation. Acta Theriologica 38, 199-232.

Quévreux, P. \& Brose, U. (2019) Metabolic adjustment enhances food web stability. Oikos 128, $54-63$.

Rinaldi, S., Muratori, S. \& Kuznetsov, Y. (1993) Multiple attractors, catastrophes and chaos in seasonally perturbed predator-prey communities. Bulletin of Mathematical Biology 55, 15-35. 
Rudolf, V.H. (2007a) Consequences of stage-structured predators: cannibalism, behavioral effects, and trophic cascades. Ecology 88, 2991-3003.

Rudolf, V.H. (2007b) The interaction of cannibalism and omnivory: consequences for community dynamics. Ecology 88, 2697-2705.

Saavedra, S., Rohr, R., Fortuna, M., Selva, N. \& Bascompte, J. (2016) Seasonal species interactions minimize the impact of species turnover on the likelihood of community persistence. Ecology $\mathbf{9 7}$, $865-873$.

Savage, V.M., Gillooly, J., Woodruff, W., West, G., Allen, A., Enquist, B.J. \& Brown, J. (2004) The predominance of quarter-power scaling in biology. Functional Ecology 18, 257-282.

Schneider, F.D., Scheu, S. \& Brose, U. (2012) Body mass constraints on feeding rates determine the consequences of predator loss. Ecology Letters 15, 436-443.

Sinclair, A.R.E. \& Arcese, P. (1995) Serengeti II: dynamics, management, and conservation of an ecosystem, vol. 2. University of Chicago Press.

Stenseth, N.C., VIljugrein, H., Jędrzejewski, W., Mysterud, A. \& Pucek, Z. (2002) Population dynamics of Clethrionomys glareolus and Apodemus flavicollis: seasonal components of density dependence and density independence. Acta Theriologica 47, 39-67.

Sunde, P., Overskaug, K. \& Kvam, T. (1999) Intraguild predation of lynxes on foxes: evidence of interference competition? Ecography 22, 521-523.

Taylor, R.A., Sherratt, J.A. \& White, A. (2013) Seasonal forcing and multi-year cycles in interacting populations: lessons from a predator-prey model. Journal of Mathematical Biology 67, 17411764. 
Thompson, R.M., Hemberg, M., Starzomski, B.M. \& Shurin, J.B. (2007) Trophic levels and trophic tangles: the prevalence of omnivory in real food webs. Ecology 88, 612-617.

Turchin, P. \& Batzli, G.O. (2001) Availability of food and the population dynamics of arvicoline rodents. Ecology 82, 1521-1534.

Turchin, P. \& Hanski, I. (1997) An empirically based model for latitudinal gradient in vole population dynamics. The American Naturalist 149, 842-874.

Ulanowicz, R.E. \& Kay, J.J. (1991) A package for the analysis of ecosystem flow networks. Environmental Software 6, 131-142.

Ushio, M., Hsieh, C.h., Masuda, R., Deyle, E.R., Ye, H., Chang, C.W., Sugihara, G. \& Kondoh, M. (2018) Fluctuating interaction network and time-varying stability of a natural fish community. Nature 554, 360 .

Weitz, J.S. \& Levin, S.A. (2006) Size and scaling of predator-prey dynamics. Ecology letters 9, $548-557$.

Williams, R.J., Brose, U. \& Martinez, N.D. (2007) Homage to Yodzis and Innes 1992: scaling up feeding-based population dynamics to complex ecological networks. From energetics to ecosystems: the dynamics and structure of ecological systems, pp. 37-51, Springer.

Yodzis, P. \& Innes, S. (1992) Body size and consumer-resource dynamics. The American Naturalist 139, 1151-1175.

Zub, K., Sönnichsen, L. \& Szafrańska, P. (2008) Habitat requirements of weasels Mustela nivalis constrain their impact on prey populations in complex ecosystems of the temperate zone. Oecologia 157, 571-582. 